\title{
A FREE-BOUNDARY PROBLEM FOR THE HEAT EQUATION ARISING IN FLAME PROPAGATION
}

\author{
LUIS A. CAFFARELLI AND JUAN L. VÁZQUEZ
}

\begin{abstract}
We introduce a new free-boundary problem for the heat equation, of interest in combustion theory. It is obtained in the description of laminar flames as an asymptotic limit for high activation energy. The problem asks for the determination of a domain in space-time, $\Omega \subset \mathbf{R}^{n} \times(0, T)$, and a function $u(x, t) \geq 0$ defined in $\Omega$, such that $u_{t}=\Delta u$ in $\Omega, u$ takes certain initial conditions, $u(x, 0)=u_{0}(x)$ for $x \in \Omega_{0}=\partial \Omega \cap\{t=0\}$, and two conditions are satisfied on the free boundary $\Gamma=\partial \Omega \cap\{t>0\}: u=0$ and $u_{\nu}=-1$, where $u_{\nu}$ denotes the derivative of $u$ along the spatial exterior normal to $\Gamma$. We approximate this problem by means of a certain regularization on the boundary and prove the existence of a weak solution under suitable assumptions on the initial data.
\end{abstract}

\section{INTRODUCTION}

In this paper we consider a new free-boundary problem for the heat equation. In classical terms it is formulated as follows: given a continuous and nonnegative initial function $u_{0}$ defined in $\mathbf{R}^{n}$, whose positivity set is an open subset $\Omega_{0}$, the problem consists in finding a domain $\Omega \subset Q_{T}=\mathbf{R}^{n} \times(0, T)$ for some $T>0$, and a smooth function $u$ in $\Omega$, such that the following conditions are met. Firstly, the heat equation is satisfied in $\Omega$ :

$$
u_{t}=\Delta u \text { in } \Omega \text {. }
$$

Secondly, the parabolic boundary $\partial_{p} \Omega$ of $\Omega$, i.e., the boundary of $\Omega$ in $\mathbf{R}^{n} \times$ $[0, T)$, consists of two pieces, namely the initial boundary $\bar{\Omega}_{0}$ (the closure of $\Omega_{0}$ in $\mathbf{R}^{n}$ ) and the lateral boundary $\Gamma=\partial \Omega \cap Q_{T}$. The solution $u$ is continuously differentiable up to $\Gamma$, also called the free boundary, where the following conditions hold:

$$
u=0 \text { and }|\nabla u|=c,
$$

where $c>0$ is a constant and $\nabla u$ denotes the gradient of $u$. Without loss of generality we will make in the sequel the assumption that $c=1$. We may also write the gradient condition in the form $u_{\nu}=-1$, where $u_{\nu}$ denotes the derivative of $u$ with respect to the outward spatial normal $\nu$ to $\Gamma$. The lateral boundary starts from the initial position $\Gamma_{0}=\partial \Omega_{0}$, where in this case

Received by the editors October 26, 1992.

1991 Mathematics Subject Classification. Primary 35K05, 35K55, 80A25.

Key words and phrases. Heat equation, free-boundary problem, combustion, regularization method, weak solutions. 
$\partial$ denotes boundary in $\mathbf{R}^{n}$. Finally, we supplement $(0.1)$ and $(0.2)$ with initial conditions

$$
u(x, 0)=u_{0}(x) \text { for } x \in \Omega_{0} .
$$

Let us call this problem (P). It appears in combustion theory in the analysis of the propagation of curved flames. It is derived in the framework of the theory of equidiffusional premixed flames analysed in the relevant limit of high activation energy, as developed for instance in Buckmaster and Ludford [BuL]. In this application $u$ has the meaning of minus temperature, precisely $u=\lambda\left(T_{c}-T\right)$, where $T_{c}$ is the flame temperature and $\lambda$ is a normalization factor. The very way the problem is derived as a simplified asymptotic model suggests viewing (P) as the limit of approximating problems $\left(\mathbf{P}_{\varepsilon}\right), \varepsilon>0$, consisting of the semilinear equation

$$
\left(\mathrm{E}_{\varepsilon}\right)=(0.4) \quad u_{t}=\Delta u-\beta_{\varepsilon}(u),
$$

where $\beta_{\varepsilon}: \mathbf{R} \rightarrow \mathbf{R}$ is $C^{\infty}$-smooth, nonnegative and bounded, with $\beta_{\varepsilon}(s)=0$ for $s \leq 0$ and support in a small neighbourhood of $s=0$. We take initial conditions

$$
u_{\varepsilon}(x, 0)=u_{0 \varepsilon}(x),
$$

where the $u_{0 \varepsilon}$ are $C^{\infty}$-smooth and nonnegative approximations of $u_{0}$. In order to approximate $(\mathbf{P})$, the regularization term $\beta_{\varepsilon}(u)$ has to satisfy certain conditions which will be discussed below. Observe that the term $\beta_{\varepsilon}(u)$ acts as an absorption term in equation (0.4). Since $T=T_{c}-(u / \lambda)$, it is in fact a reaction term for the temperature. In the flame model such a term represents the effect of the exothermic chemical reaction.

An important question in the application is the convergence of solutions to travelling waves, which leads to the study of a stationary free-boundary problem. This question has been discussed for equations like (0.4) in a series of recent papers by Berestycki and coauthors, cf. [BL], [BLN], [BLL], [BCN]. On the other hand, a similar evolution problem appears in the study of detonation waves as proposed in Stewart [S] and Stewart and Ludford [SL]. It leads to Burger's equation $u_{t}=u_{x x}+u u_{x}$, under lateral conditions like (0.2). The stability of travelling waves for this one-dimensional problem has been studied by Brauner, Lunardi, and Schmidt-Lainé in [BLS]. These papers contain extensive references to related combustion literature. Other works on this problem will be commented on at the end of the introduction.

Let us go back to the solution of $(\mathrm{P})$ by means of the regularization method. Problem $\left(\mathbf{P}_{\varepsilon}\right)$ admits a unique classical solution $u_{\varepsilon} \in C^{\infty}(Q), Q=\mathbf{R}^{n} \times$ $(0, \infty)$, which is positive everywhere in $Q$. The Maximum Principle holds. We want $\left(\mathbf{P}_{\varepsilon}\right)$ to approximate $(\mathbf{P})$ as $\varepsilon \rightarrow 0$ in a weak sense. A natural weak formulation for problem (P) asks for a domain $\Omega$ with Lipschitz continuous lateral boundary $\Gamma$ and a function $u \in C(\Omega \cup \Gamma)$ such that:

(i) for every test function $\phi \in C_{0}^{\infty}\left(\mathbf{R}^{n} \times[0, T)\right)$

$$
\iint_{\Omega} u\left(\phi_{t}+\Delta \phi\right) d x d t+\int_{\Omega_{0}} u_{0} \phi d x=\int_{\Gamma} \phi d \Sigma \cos \alpha,
$$

(ii) $u$ vanishes on $\Gamma$, and 
(iii) the free boundary $\Gamma$ starts from $\Gamma_{0}=\partial \Omega_{0}$, i.e., the section $\Gamma_{t}$ at time $t$ converges to $\Gamma_{0}$ as $t \rightarrow 0$ in some sense.

In (0.6) $d \Sigma$ is the area element on $\Gamma$ and $\alpha$ is the angle formed by the exterior normal $\nu(x, t)$ at a point $(x, t) \in \Gamma$ and the hyperplane $t=$ constant, so that $d S=d \Sigma \cos \alpha$ is the lateral projection of the element $d \Sigma$.

In order for $\left(\mathbf{P}_{\varepsilon}\right)$ to approximate problem $(\mathbf{P})$ we need to impose some conditions both on the initial data $u_{0 \varepsilon}$ and on the absorption functions $\beta_{\varepsilon}$. One of the difficulties we face consists in finding a solution with a free boundary $\Gamma$ which starts from the initial boundary $\Gamma_{0}$. Now, if for example we take compactly supported initial data $u_{0}$, functions $\beta_{\varepsilon}$ with support in the interval $[0, \varepsilon]$, and approximations $u_{0 \varepsilon}$ to the data such that $u_{0 \varepsilon} \geq \varepsilon$, it clearly follows that the absorption term has no effect and we obtain just positive solutions of the heat equation in $Q$ with no free boundary.

Such a difficulty has already been studied in the stationary case by Berestycki, Caffarelli, and Nirenberg [BCN], which find convenient conditions on the absorption functions. Roughly speaking, $\beta_{\varepsilon}(s)$ has to be concentrated in a right neighbourhood of $s=0$ and its mass $M=\int \beta_{\varepsilon}(s) d s$ has to be directly related to the value $u_{\nu}=-1$ that we seek to obtain in the limit on the free boundary. The precise assumptions will be the following. We define the family $\beta_{\varepsilon}$ in terms of a single function $\beta$ by

$$
\beta_{\varepsilon}(s)=\frac{1}{\varepsilon} \beta\left(\frac{s}{\varepsilon}\right) .
$$

This is very convenient in order to use scaling arguments. We will assume that the function $\beta: \mathbf{R} \rightarrow \mathbf{R}$ satisfies the following assumptions:

(i) $\beta$ is positive in the interval $I=\{0<s<1\}$ and 0 otherwise,

(ii) it is a $C^{\infty}$ function in $[0, \infty)$,

(iii) it is increasing for $0 \leq s<1 / 2$, decreasing for $1 / 2<s \leq 1$,

(iv) the integral of $\beta, \int \beta(s) d s$, equals $1 / 2$.

See also (4.1).

As we have said, obtaining a solution with the correct free boundary depends also on the way the initial data are approximated. As already pointed out in $[\mathrm{BCN}]$ in the elliptic case, we need to have approximations on which the whole force of $\beta_{\varepsilon}$ acts. The simplest way is asking not only that the $u_{0 \varepsilon}$ uniformly approximate $u_{0}$ but also that their supports converge to $\Omega_{0}$ in the usual metric for subsets of $\mathbf{R}^{n}$, but see Section 8 .

We begin the study of the existence of weak solutions with the existence of self-similar solutions of the form $u(x, t)=(T-t)^{1 / 2} f\left(x /(T-t)^{1 / 2}\right)$. We construct for every $T>0$ one such solution with compact support, which solves $(\mathbf{P})$ in the classical sense and vanishes identically at time $T$. We also prove that such a solution is the unique limit of the solutions of suitable approximate problems $\left(\mathbf{P}_{\varepsilon}\right)$. Moreover, a stability result is established. It is interesting to remark that on the free boundary

$$
\Gamma=\left\{(x, t): 0 \leq t \leq T,|x|^{2}=R^{2}(T-t)\right\}
$$

we have not only the boundary condition $u_{\nu}\left(=u_{r}\right)=-1$ but also a dynamic equation for the movement of the free boundary. Formally, this equation is derived by differentiating along a normal spatial direction the equation $u(x, t)=0$ which holds at the free boundary. If the free boundary moves along such a direction with velocity $\mathbf{V}$ we get $\nabla u \cdot \mathbf{V}+u_{t}=0$, from which, thanks to the 
boundary condition, it follows that

$$
\mathbf{V}=u_{t} \mathbf{n}=\Delta u \mathbf{n}
$$

(where $\mathbf{n}=\mathbf{n}(x, t) \in \mathbf{R}^{n}$ denotes the exterior spatial normal vector to the free boundary, and $u_{t}$ and $\Delta u$ are understood as limits approaching the boundary from the region $\{u>0\}$ ). Such an equation is satisfied by the self-similar solutions. The study is completed in Section 2 with a similar analysis of existence of travelling waves and solutions with holes.

We then proceed with the study of $(\mathbf{P})$ for general data. We assume throughout that $u_{0}$ is bounded, nonnegative, and Lipschitz continuous. In Section 3 we obtain integral a priori bounds of classical type. Section 4 establishes the important gradient bound by means of a technique of Bernstein type. Section 5 shows how to obtain the same bound for $t>0$ without assuming that it holds at $t=0$. Section 6 derives continuity in $t$. With these results we may pass to the limit $\varepsilon \rightarrow 0$ and obtain in Section 7 a continuous and nonnegative function $u$ which satisfies the heat equation wherever it is positive.

The study of the boundary behaviour of the limit function is more delicate and is taken up in the remaining sections. We have chosen to consider a natural situation for the application in mind in which the solution has $u_{t} \leq 0$ and thus shrinking support; in other words, the flame advances. A precise formulation of the convenient hypotheses is given in Section 8. Such an assumption very much simplifes the boundary analysis since it implies, to begin with, that the free boundary is a Lipschitz continuous surface. Section 9 begins the analysis of the boundary measure, while Section 10 discusses the behaviour at regular points of the free boundary. We obtain in particular the dynamic equation (0.8) with derivatives taken along nontangential interior directions. Finally, in Section 11 the proof of existence of a weak solution is completed. The main result we obtain can be stated as follows.

Theorem. Assume that $u_{0}$ is a nonnegative, Lipschitz continuous, and bounded function in $\mathbf{R}^{n}$ satisfying moreover the precise conditions stated in assumptions $(\mathrm{H} 1)$ and $(\mathrm{H} 2)$, Section 8 . Then there exists a weak solution of problem $(\mathbf{P})$. If $u_{0}$ has compact support then the solution vanishes in finite time.

We have chosen to consider the simplest equation corresponding to this kind of problem. Our results admit of course natural extensions to equations of the form

$$
u_{t}=\sum a_{i j}(x, t) \frac{\partial^{2} u}{\partial x_{i} \partial x_{j}},
$$

or equations with lower-order terms. We then replace the boundary condition by

$$
\sum a_{i j} \partial_{i} u \partial_{j} u=1 \text { on } \Gamma \text {. }
$$

Moreover, typical flame problems are considered in cylindrical spatial domains of the form $S=\mathbf{R} \times D$, where $D$ is a bounded domain in $\mathbf{R}^{n-1}$ with smooth boundary. On the fixed boundary of the 'tube' $S$ we impose Neumann data $u_{\nu}=0$. We refer to [BCN] for more details about the elliptic case.

We do not discuss here the question of uniqueness but for the case of the special solutions constructed in Section 1 (such a result is then used in Section 
6). The question of uniqueness in the general framework seems to be a difficult one. There are however results in one space dimension for the Burger's problem $u_{t}=u_{x x}+u u_{x}$ mentioned above. An existence and uniqueness theory is developed in $[\mathrm{HH}]$ for a class of nonnegative solutions which converge to travelling waves. The method is based on a modification of the equation into a mixed elliptic-parabolic problem. It applies also to the heat equation case. A two-phase problem is treated in [BHS] by means of a regularization of the jump at the interface, say $u=0$, of the form $H(u)_{x}$, where $H$ is the Heaviside function. However, these approaches, which adapt well to the one-dimensional situation, do not seem to apply for higher space dimensions.

\section{A SPECIAL SELF-Similar SOlUtion}

We construct in this section a particular family of classical solutions of our problem, which will be important in understanding what we expect from the general theory in the best of cases and in providing us with a useful tool in comparison arguments. We will show the existence and uniqueness of such solutions as well as their stability.

1.1. Existence. We are looking for a solution in the self-similar form

$$
U(x, t)=(T-t)^{\alpha} f\left(x /(T-t)^{\beta}\right) .
$$

Plugging this form into equation (0.1) and boundary conditions $(0.2)$, we see that necessarily $\alpha=\beta=1 / 2$, while $f=f(\xi), \xi=x(T-t)^{-1 / 2}$, solves

$$
\Delta f=-\frac{1}{2} f+\frac{1}{2} \xi \cdot \nabla f \text { where } f>0,
$$

with boundary conditions $f=0$ and $f_{\nu}=-1$. Assuming also that $f$ is radially symmetric, i.e., $f=f(r)$ with $r=|x|(T-t)^{-1 / 2} \geq 0$, we get for $f$ the following problem.

(E) To find a number $R>0$ and a $C^{1}$ function $f(r)$, defined for $0 \leq r \leq R$ and such that

$$
\begin{aligned}
& f^{\prime \prime}+\left\{\frac{n-1}{r}-\frac{1}{2} r\right\} f^{\prime}+\frac{1}{2} f=0 \text { for } 0<r<R, \\
& f^{\prime}(0)=0 \text { and } f(r)>0 \text { for } 0 \leq r<R, \\
& f(R)=0 \text { and } f^{\prime}(R)=-1 .
\end{aligned}
$$

(The superscript ' denotes derivative with respect to $r$.) Then we have

Proposition 1.1. There exists a unique solution of problem (E).

Proof. We will use a shooting argument. We solve equation (1.3a) with initial data

$$
f(0)=1, \quad f^{\prime}(0)=0 .
$$

Clearly a solution exists and is positive in a certain interval $\left(0, r_{0}\right)$. Let us show that this interval can be continued until $f$ has a zero, which we will denote by $R$. In fact, it follows from $(1.3 a)$ that $f^{\prime \prime}(r) \approx-1 / 2 n$ for $r \approx 0$. This means that $f$ will be decreasing for $r \approx 0$. Next, we observe that $f^{\prime}$ will 
be negative as long as $f>0$. (Hint: Argue by contradiction at the first point where $f^{\prime}=0$.) Moreover, in the interval $0<r<R$ we have

$$
\left(r^{n-1} f^{\prime}(r)\right)^{\prime}=\frac{1}{2} r^{n-1}\left\{r f^{\prime}(r)-f\right\} .
$$

Therefore, the quantity $r^{n-1} f^{\prime}(r)$ is decreasing in $r$ if $0<r<R$. Let us prove next that $R$ is finite. Otherwise, $f$ would be defined and positive for all $r>0$. Now, for $r^{2}>2(n-1)$ we would have, from equation (1.3a), $f^{\prime \prime}(r)<-\frac{1}{2} f(r)<0$. Hence $f$ would be concave and decreasing. Hence, it must have a finite zero.

Finally, we observe that the monotonicity of $r^{n-1} f^{\prime}(r)$ implies that $f^{\prime}(R)<$ 0 . Let us put $c=-f^{\prime}(R)>0$. Since equation (1.3.a) is linear, if we want to achieve the boundary condition $f^{\prime}(R)=-1$ we only need to multiply $f$ by $1 / c$, i.e., pick $f(0)=1 / c$ as shooting height. This also shows uniqueness and completes the proof.

Remarks. Here are some of the properties of the self-similar solution just constructed, that we will denote in the sequel for convenience as $U(x, t ; T)$.

(1) In one spatial dimension $f$ is given by integration of the formula

$$
f^{\prime \prime}(r)=-\frac{a}{2} e^{\frac{r^{2}}{4}}
$$

with $f(0)=a, f^{\prime}(0)=0$, and $a$ properly chosen. Note that $f^{\prime \prime}(r) \leq-a / 2$.

(2) $U$ has initial values $U(x, 0)=T^{\frac{1}{2}} f\left(x / T^{\frac{1}{2}}\right)$, so that $\sup (U(x, 0))=$ $a T^{\frac{1}{2}}$. The solution is $C^{\infty}$-smooth in the support

$$
\bar{\Omega}=\left\{(x, t): 0 \leq t \leq T, \quad|x| \leq R(T-t)^{\frac{1}{2}}\right\}
$$

for $0<t<T$. Therefore, $U$ is a classical solution of $(\mathrm{P})$ in $Q_{T}$. However, at the extinction point, $x=0, t=T$, the free boundary

$$
|x|=R(T-t)^{\frac{1}{2}}
$$

becomes horizontal and the regularity in $t$ is of Hölder type $C^{\frac{1}{2}}$, namely $U$ decays as $t \rightarrow T$ as $O\left((T-t)^{\frac{1}{2}}\right)$.

(3) It can be easily checked by differentiation of (1.3a) that $f^{\prime}$ cannot have a minimum at a point where $f>0$. Therefore, $\left|f^{\prime}(r)\right| \leq 1$ and $|\nabla U(x, t)| \leq 1$ in the support of the solution. At the extinction point the spatial gradient is not defined; the nontangential limit of $|\nabla U|$ is zero, while the limit along the free surface is of course 1 .

1.2. Limits, uniqueness, and stability. One of the aims of this work is to prove that the problems $\left(\mathbf{P}_{\varepsilon}\right)$ do approximate the (weak) solution of problem $(\mathbf{P})$. We are going to prove next that this is true for the present initial data. More precisely we will show that in the limit we always get the classical solution $U$ just described.

Proposition 1.2. The self-similar solution $U=U(x, t ; T)$ is the unique limit of the approximation process $\left(\mathbf{P}_{\varepsilon}\right)$ corresponding to initial data $U(x, 0 ; T)$, and this happens for any admissible functions $\beta_{\varepsilon}$, and any $u_{0 \varepsilon}$ which approximate $u_{0}$ uniformly and such that $\operatorname{supp}\left(u_{0 \varepsilon}\right) \rightarrow \operatorname{supp}\left(u_{0}\right)$ uniformly.

Proof. The proof relies on the construction of suitable super- and subsolutions for the problems $\left(\mathbf{P}_{\varepsilon}\right)$ which in the limit $\varepsilon \rightarrow 0$ force the approximate solutions $u_{\varepsilon}$ to converge to $U$. 
Step 1. First, we observe that a rescaling of the form

$$
U^{\lambda}(x, t)=U\left(\frac{x}{\lambda}, \frac{t}{\lambda^{2}}\right), \quad \lambda>0,
$$

gives a new solution of the heat equation in the domain

$$
\Omega^{\lambda}=\left\{(x, t): 0<t<\lambda^{2} T,|x|<R\left(\lambda^{2} T-t\right)^{1 / 2}\right\},
$$

satisfying the boundary condition

$$
\frac{\partial U^{\lambda}}{\partial \nu}=-\frac{1}{\lambda}
$$

We have

$$
U^{\lambda}(x, t ; T)=\frac{1}{\lambda} U\left(x, t ; \lambda^{2} T\right)=\frac{1}{\lambda}\left(\lambda^{2} T-t\right)^{1 / 2} f\left(\frac{x}{\left(\lambda^{2} T-t\right)^{1 / 2}}\right) .
$$

The extinction time for $U^{\lambda}$ is therefore $T_{\lambda}=\lambda^{2} T$. We see that for $0<$ $\lambda<1$ the initial data decrease (though the sup-norm is conserved), the domain contracts in $x$ and $t$ and the flux through the boundary increases. We have all the ingredients of a subsolution. On the other hand, for $\lambda>1$ the above effects are reversed and we obtain a candidate supersolution. In order to substantiate such claims we prove next that small perturbations of these functions are in fact super- and subsolutions for the approximate problems. Notice that the Maximum Principle applies to equation $\left(\mathrm{E}_{\varepsilon}\right)$.

Step 2. Let us deal with the subsolution. We start with a rescaled function $\tilde{U}(x, t)=U^{\lambda}(x, t ; T)$ with a fixed $\lambda<1, \lambda \approx 1$. It has the self-similar form (1.1) with extinction time $T_{\lambda}=\lambda^{2} T<T$ and profile

$$
\tilde{f}(\xi)=\frac{1}{\lambda} f(\xi) \geq f(\xi) \text {. }
$$

We will construct a subsolution $\tilde{u}_{\varepsilon}$ of $\left(\mathbf{P}_{\varepsilon}\right)$ as a small perturbation of $\tilde{U}$. It will have the same form

$$
\tilde{u}_{\varepsilon}(x, t)=\left(T_{\lambda}-t\right)^{\frac{1}{2}} f_{\varepsilon}\left(x /\left(T_{\lambda}-t\right)^{\frac{1}{2}}\right) .
$$

In order to compare $\tilde{u}_{\varepsilon}$ with the actual approximation $u_{\varepsilon}$ we have to check the subsolution property both on the equation and the initial data. The condition of subsolution of $\left(\mathrm{E}_{\varepsilon}\right)$ means that we have to ask that

$$
f_{\varepsilon}^{\prime \prime}+\left\{\frac{n-1}{r}-\frac{r}{2}\right\} f_{\varepsilon}^{\prime}+\frac{1}{2} f_{\varepsilon} \geq\left(T_{\lambda}-t\right)^{\frac{1}{2}} \beta_{\varepsilon}\left(\left(T_{\lambda}-t\right)^{\frac{1}{2}} f_{\varepsilon}\right) .
$$

In view of this we decide to consider a time interval $0<t<h T_{\lambda}$ with $h>0$ small, and construct $f_{\varepsilon}$ as a solution of the equation

$$
f^{\prime \prime}+\left\{\frac{n-1}{r}-\frac{r}{2}\right\} f^{\prime}+\frac{1}{2} f=\mu T_{\lambda}^{\frac{1}{2}} \gamma_{\varepsilon}\left(T_{\lambda}^{\frac{1}{2}} f\right),
$$

where the new absorption function $\gamma_{\varepsilon}$ and the constant $\mu=\mu_{\varepsilon}>0$ are to be determined. Comparison of (1.12) and (1.13) suggests that (1.12) is satisfied if we take

$$
\mu \geq 1,
$$


and $\gamma_{\varepsilon}$ is chosen so as to satisfy the following condition: for every $s>0$ and $(1-h)^{\frac{1}{2}} \leq \theta \leq 1$ we have

$$
\gamma_{\varepsilon}(s) \geq \beta_{\varepsilon}(\theta s) .
$$

Copying from (0.7) we can obtain all the $\gamma_{\varepsilon}$ 's from a single $\gamma$ by putting $\gamma_{\varepsilon}(s)=\varepsilon^{-1} \gamma\left(\varepsilon^{-1} s\right)$. We can easily construct a $\gamma$ satisfying (1.15). It will be supported in the interval $[0, l]$ with $l=1 /(1-h)^{\frac{1}{2}}$ and will have an integral

$$
M=\int \gamma(s) d s>\frac{1}{2},
$$

which can be made close to $1 / 2$ by taking $h$ small. We will also need $\gamma(s) \geq \alpha s$ for some small $\alpha$ and all $0<s<1 / 2$ for technical reasons.

Going back to the construction of $f_{\varepsilon}$, we impose initial data at $r=0$ equal to $\tilde{f}$, namely

$$
f_{\varepsilon}(0)=\frac{a}{\lambda} \quad \text { and } \quad f_{\varepsilon}^{\prime}(0)=0 .
$$

It is clear that as long as $T_{\lambda}^{1 / 2} f_{\varepsilon} \geq l \varepsilon$ the functions $\tilde{f}$ and $f_{\varepsilon}$ coincide. We need to select the constant $\mu=\mu_{\varepsilon}$ in such a way that the function $f_{\varepsilon}$ ends up nicely at the level $f=0$. In this respect we have the following precise result.

Lemma 1.3. Let $f_{\varepsilon}$ be the solution of (1.13) with initial data (1.17). There exists one value of the constant $\mu$, say $\mu_{0}(\varepsilon)$, such that for $\mu<\mu_{0}(\varepsilon)$ the solution crosses the $(f=0)$-axis at some point $R_{1}>R$, while for $\mu=\mu_{0}(\varepsilon)$ it is positive and goes to zero as $r \rightarrow \infty$. Moreover, as $\varepsilon \rightarrow 0$ we have, with the above notations,

$$
\mu_{0}(\varepsilon) \rightarrow \mu_{*}=\frac{1}{2 M \lambda^{2}}
$$

( $\mu_{0}$ will depend in fact on $\varepsilon, \lambda$, and $M$ ). We can estimate $R<R_{1}<R+C \varepsilon$ if $\mu \leq \mu_{0}, \quad \mu \approx \mu_{0}$.

Proof. (i) We take $\varepsilon$ small enough and start the construction of $f_{\varepsilon}$ from the initial values (1.17). As long as $T_{\lambda}^{1 / 2} f_{\varepsilon} \geq l \varepsilon$ the solution $f_{\varepsilon}$ coincides with $\tilde{f}$; hence it is monotonically decreasing. There is a point $r_{\varepsilon}$ at which the level $c_{\varepsilon}=T_{\lambda}^{-1 / 2} l \varepsilon$ is attained, and clearly there $f_{\varepsilon}^{\prime}\left(r_{\varepsilon}\right) \approx-1 / \lambda$. The point $r_{\varepsilon}$ is thus estimated in first approximation as $r_{\varepsilon} \approx R-T^{-1 / 2} l \varepsilon$.

From this point on and as long as $f_{\varepsilon}$ takes values in the interval $\left(0, c_{\varepsilon}\right)$, the effect of the second member of equation (1.13) will tend to make the derivative $f_{\varepsilon}$ less negative. We can calculate this effect by multiplying (1.13) by $f_{\varepsilon}^{\prime}$ and integrating from $r_{\varepsilon}$. We thus obtain for $r>r_{\varepsilon}$

$$
\frac{1}{2}\left(f_{\varepsilon}^{\prime}(r)\right)^{2}-\frac{1}{2}\left(f_{\varepsilon}^{\prime}\left(r_{\varepsilon}\right)\right)^{2}=\mu\left\{G_{\varepsilon}\left(T_{\lambda}^{1 / 2} f_{\varepsilon}(r)\right)-M\right\}+I_{\varepsilon},
$$

where $G(s)=\int_{0}^{s} \gamma(s) d s$ and

$$
I_{\varepsilon}=\int_{r_{\varepsilon}}^{r}\left\{\frac{r}{2}-\frac{n-1}{r}\right\}\left(f_{\varepsilon}^{\prime}\right)^{2} d r+\frac{1}{4}\left\{f_{\varepsilon}^{2}\left(r_{\varepsilon}\right)-f_{\varepsilon}^{2}(r)\right\},
$$

which will be of the order of $r-r_{\varepsilon}$ for small $\varepsilon$. We conclude that as long as $r-R=O(\varepsilon)$ we have but for terms of the order of $\varepsilon$

$$
\left(f_{\varepsilon}^{\prime}(r)\right)^{2} \approx\left(\frac{1}{\lambda^{2}}-2 M \mu\right)+2 \mu G_{\varepsilon}\left(T_{\lambda}^{1 / 2} f_{\varepsilon}(r)\right)
$$


We now fix a value of $\mu$ less than $\mu_{*}$, say $\mu<\mu_{*}-a$. Then for $\varepsilon$ small enough we conclude form (1.20) that the function $f_{\varepsilon}$ gets from the level $c_{\varepsilon}$ at $r_{\varepsilon}$ to the level $f_{\varepsilon}=0$ at some point $R_{1}=R_{1}(\mu, \varepsilon)$ with an always negative slope, and

$$
\left(f_{\varepsilon}^{\prime}\left(R_{1}\right)\right)^{2} \approx 2 M\left(\mu_{*}-\mu\right) \text {. }
$$

From this and the value of $f_{\varepsilon}\left(r_{\varepsilon}\right)$ we get the estimate

$$
R_{1}-r_{\varepsilon} \leq \varepsilon l T^{-1 / 2}\left(1-\left(\mu / \mu_{*}\right)\right)^{-1 / 2}+o(\varepsilon) \leq \varepsilon l\left(\mu_{*} / a T\right)^{1 / 2} .
$$

(ii) On the other hand, given $a>0$, if $\mu>\mu_{*}+a$ and $\varepsilon$ small enough, then the solution $f_{\varepsilon}$ has a positive minimum at a point near $R$ after which it turns up until it leaves the region $\left(0, c_{\varepsilon}\right)$. In justifying this assertion we need the zero-order term of (1.13), namely $\frac{1}{2} f-\mu T_{\lambda}^{1 / 2} \gamma_{\varepsilon}\left(T_{\lambda}^{1 / 2} f\right)$, to be nonpositive, which holds if we impose the condition $\gamma(s) \geq \alpha s$ for some $\alpha>0$ and $s \approx 0$.

(iii) In order to obtain the value $\mu_{0}(\varepsilon)$ we first observe that among the solutions described in (i) there is a natural order, i.e., $f_{\varepsilon}$ increases as $\mu$ increases. Therefore, we take $\mu_{0}$ to be the supremum of the values of $\mu$ for which we obtain a solution $f_{\varepsilon}$ which crosses the range of values $\left(0, c_{\varepsilon}\right)$ in the way described in (i). We clearly have $\mu_{*}-a \leq \mu_{0} \leq \mu_{*}+a$ if $\varepsilon$ is small. By standard arguments in ODE theory we conclude that the limit solution, i.e., the supremum of the previous family, has to be decreasing for $r>r_{\varepsilon}$, positive, and has to tend to 0 as $r \rightarrow \infty$. This proves (1.18).

It follows that the right choice of $\mu$ in the construction (1.11), (1.13) will be

$$
\mu<\mu_{0}(\varepsilon) \text {. }
$$

In view of (1.18) and the observation after (1.16), the two conditions on $\mu_{\varepsilon}$, (1.14) and (1.21), can be clearly met if $h$ is small enough, $h \leq h(\lambda)$, and $\varepsilon$ is small. Notice that $h$ does not depend on $T$.

We must also check the initial data. It can happen that $\tilde{u}_{\varepsilon}(x, 0)$ is smaller than $u_{0 \varepsilon}(x)$, for instance if the approximation $u_{0 \varepsilon}$ is taken to be larger than $U_{0}$. Otherwise we have to replace formula (1.11) by

$$
\tilde{u}_{\varepsilon}(x, t)=\left(T_{\lambda}-t-\delta\right)^{1 / 2} f_{\varepsilon}\left(x /\left(T_{\lambda}-t-\delta\right)^{1 / 2}\right),
$$

for some small $\delta>0$ related to $\varepsilon$. This minor modification does not alter the fact that $\tilde{u}_{\varepsilon}$ is a subsolution for equation $\left(\mathrm{E}_{\varepsilon}\right)$. In this way we arrive at the conclusion that small perturbations of $\tilde{U}$ are subsolutions for all the problems $\left(\mathrm{P}_{\varepsilon}\right)$. It follows that in the limit $\varepsilon \rightarrow 0$ of the actual solutions $u_{\varepsilon}$ we get

$$
U^{\lambda}(x, t) \leq \liminf _{\varepsilon \rightarrow 0} u_{\varepsilon}(x, t)
$$

for every $x \in \mathbf{R}^{n}$ and every $0 \leq t \leq h T$.

Step 3. The proof proceeds by running again the same method. Observe that $\tilde{U}\left(x, h T_{\lambda}\right)$ is the same kind of initial data as $\tilde{U}(x, 0)$ since

$$
\tilde{U}\left(x, h T_{\lambda}\right)=U^{\lambda}\left(x, h T_{\lambda} ; T\right)=U^{\lambda}(x, 0 ; T(1-h)) .
$$

Therefore, the step of approximation can be repeated up to a second time $t_{2}=$ $h T_{\lambda}+h T_{\lambda}(1-h)$ (taking care of inserting an extra $\delta$ to fit the initial conditions). And the process continues with subsequent steps. We conclude that we can reach 
with estimate (1.23) up to any time less that $T_{\lambda}$. We can now let $\lambda \rightarrow 1$ and obtain that in $0 \leq t \leq T$

$$
U(x, t) \leq \liminf _{\varepsilon \rightarrow 0} u_{\varepsilon}(x, t) .
$$

Step 4. A similar construction applies from above with $\lambda>1, \lambda \rightarrow 1$ to show that

$$
U(x, t) \geq \limsup _{\varepsilon \rightarrow 0} u_{\varepsilon}(x, t)
$$

holds for $t>0$. Now we have to use the solution for $\mu_{0}(\varepsilon)$ to construct a supersolution.

An immediate and very useful consequence of the above result is the possibility of using comparison arguments involving the self-similar solutions.

Corollary 1.4. The Maximum Principle applies to a pair of limit solutions whenever one of them is $U(x, t ; T)$.

The proof consists simply in recalling that the Maximum Principle is true for the approximate problems, and using Proposition 1.2.

We end the study with two interesting consequences of these facts.

Proposition 1.5. The solutions $U(x, t ; T)$ are stable in our topology.

Proof. Take one of these solutions, say for a given $T>0$. Given $\delta>0$, any data $u_{0}$ whose distance from $U(x, 0 ; T)$ is less than $\delta$ can be squeezed between $U(x, t ; T+\tau)$ and $U(x, t ; T-\tau)$ for some small $\tau>0$ depending on $\delta$.

Theorem 1.6. Let $u_{0} \in C^{1}\left(\mathbf{R}^{n}\right)$ be a nonnegative function with compact support and let $u$ be obtained as the limit of the approximate problems $\left(\mathbf{P}_{\varepsilon}\right)$ with suitable $u_{0 \varepsilon}$. Then for every $t>0$ the function $u(\cdot, t)$ has compact support. Moreover, the supports are localized in a fixed ball of $\mathbf{R}^{n}$ and the solution vanishes identically in a finite time.

Proof. We bound our data from above by a suitable radially-symmetric $U(x, 0)$ given by (1.1) and apply the Maximum Principle (which, being valid for every $\varepsilon>0$, holds in the limit).

\section{OTHER SPECIAL SOLUTIONS}

2.1. Travelling waves. We consider now solutions of Problem (P) of the form

$$
u(x, t)=f\left(x_{1}-c t\right),
$$

where $x=\left(x_{1}, \ldots, x_{n}\right)$ and $c$ is a constant, the speed of the travelling wave. Let us fix the initial boundary at $\Pi_{0}=\left\{x: x_{1}=0\right\}$. Then for $c=0$ we get the two stationary profiles $f_{1}(\xi)=\max \left\{x_{1}, 0\right\}$ and $f_{2}(\xi)=\max \left\{-x_{1}, 0\right\}$. For $c>0$ there is exactly one advancing travelling wave with initial boundary $\Pi_{0}$. By advancing we mean that the domain expands. It is defined in the domain $\left\{(x, t): x_{1}<c t\right\}$ by the formula

$$
f_{c}^{a}(\xi)=\frac{1}{c}\left\{e^{-c \xi}-1\right\}
$$


with $\xi=x_{1}-c t$. There is also a receding wave (i.e., with shrinking domain) with the same boundary, defined in $\left\{(x, t): x_{1}>c t\right\}$ by

$$
f_{c}^{r}(\xi)=\frac{1}{c}\left\{1-e^{-c \xi}\right\} .
$$

Symmetry in $x_{1}$ produces solutions of the form $u(x, t)=f\left(x_{1}+c t\right)$ moving with speed $c>0$ in the direction of the negative $x_{1}$-axis. Translations in $x$ or $t$ and rotations in $x$ produce a whole set of travelling waves having as initial boundary any desired hyperplane $\Pi \in \mathbf{R}^{n}$ and moving with speed $c$ in the direction of the normal to $\Pi$. These families are completed by the stationary solutions

$$
u(x, t)=f(x), \quad \text { with } f(x)=\left[x_{1}\right]_{+} \text {or } f(x)=\left[-x_{1}\right]_{+},
$$

corresponding to the limit $c \rightarrow 0$.

It is interesting to remark that advancing travelling waves have convex profiles. Moreover,

$$
f_{c}^{a}(\xi) \approx \frac{1}{c} e^{c|\xi|} \text { as } \quad \xi \rightarrow-\infty .
$$

On the other hand, receding waves are concave and bounded and

$$
f_{c}^{r}(\xi) \rightarrow \frac{1}{c} \text { as } \xi \rightarrow \infty,
$$

while the stationary solutions are piecewise linear.

The methods of Section 1 can be used to show the uniqueness and stability of these solutions.

2.2. Stationary solutions with a hole. Assume that $n>1$. We have to find a number $R>0$ and a function $u(x)$ defined for $x>R$ such that

$$
\Delta u=0 \text { for }|x|>R, \quad u(R)=0, \quad u_{\nu}(R)=-1 .
$$

In two space dimensions, $n=2$, such a solution takes the form

$$
u(r)=R \log (r / R), \quad r=|x| .
$$

Suppose now that we fix at $r=1$ the value of the solution, say $u(1)=A$. Then, $R$ is determined by the equation

$$
A=-R \log R \text {. }
$$

This equation does not admit a solution if $A>A_{*}=1 / e$, it has a unique solution $R=1 / e$ if $A=A_{*}$, and it has two solutions if $0<A<A_{*}$, corresponding to a big hole and a small hole. Moreover, the radius of the small hole decreases to 0 as $A \rightarrow 0$ while the radius of the large hole goes to 1 . The small holes show that we can have stationary solutions as close to 0 as we like in a certain region having holes of small radius.

A similar situation happens in $n \geq 3$. Now the solution with hole of radius $R>0$ takes the form

$$
u(r)=\frac{R^{n-1}}{n-2}\left\{\frac{1}{R^{n-2}}-\frac{1}{r^{n-2}}\right\} .
$$


Observe that the solution is now bounded in its domain $\{|x|>R\}$. This is the famous Zel'dovich flame (with $n=3$, cf. [ZBLM]), which is unstable.

\section{Integral estimates. Finite propagation}

In this section we present some estimates for the solutions of Problem (P) obtained from the approximate problems $\left(\mathbf{P}_{\varepsilon}\right)$. Here and in the sequel we assume that the initial data $u_{0}$ are bounded, and that they are approximated uniformly by smooth functions $u_{0 \varepsilon}$. We have

(i) To begin with, it is clear that solutions of the heat equation $u_{t}=\Delta u$ in $Q_{T}$ with the same initial data are supersolutions for our problem. Thus, whenever $u_{0}, u_{0 \varepsilon} \in L^{p}\left(\mathbf{R}^{n}\right), 1 \leq p \leq \infty$, we get estimates of the form

$$
u_{\varepsilon}(x, t) \leq C\left\|u_{0 \varepsilon}\right\|_{p} t^{-n / 2 p},
$$

where $\|\cdot\|_{p}$ denotes the $L^{p}$-norm in $\mathbf{R}^{n}$. Since $C$ does not depend on $\varepsilon$ the same decay rate is valid in the limit $\varepsilon \rightarrow 0$.

(ii) More precisely, if $u_{0} \in L^{1}\left(\mathbf{R}^{n}\right)$ we get

$$
\int u_{\varepsilon}(x, t) d x+\iint \beta_{\varepsilon}\left(u_{\varepsilon}\right) d x d t=\int u_{0 \varepsilon}(x) d x .
$$

We thus obtain a uniform control of $\beta_{\varepsilon}\left(u_{\varepsilon}\right)$ in $L^{1}\left(Q_{T}\right)$. A local version of this estimate can be obtained as follows: Let $\zeta$ be a spatial cutoff function $\zeta(x) \in C_{0}^{\infty}\left(\mathbf{R}^{n}\right)$. Then

$$
\begin{aligned}
& \int u_{\varepsilon}(x, t) \zeta(x) d x+\iint \beta_{\varepsilon}\left(u_{\varepsilon}\right) \zeta(x) d x d t \\
& =\int u_{0 \varepsilon}(x) \zeta(x) d x+\iint u_{\varepsilon}(x, t) \Delta \zeta(x) d x d t,
\end{aligned}
$$

and the last integral is controlled since $u_{\varepsilon}$ is uniformly bounded.

(iii) Multiplication of equation (0.4) by $u_{\varepsilon}$ and integration by parts gives an $L^{2}$-estimate of the spatial gradient $\nabla u_{\varepsilon}$, which is uniform in $\varepsilon$, when $u_{0} \in$ $L^{2}\left(\mathbf{R}^{n}\right)$

$$
\frac{1}{2} \int u_{\varepsilon}^{2}(t) d x+\iint\left|\nabla u_{\varepsilon}\right|^{2} d x d t+\iint \beta_{\varepsilon}\left(u_{\varepsilon}\right) u_{\varepsilon} d x d t=\frac{1}{2} \int u_{0 \varepsilon}^{2} d x .
$$

We can also get a local version, where $\left|\nabla u_{\varepsilon}\right|$ is controlled in $L_{\text {loc }}^{2}(Q)$ :

$$
\begin{aligned}
& \frac{1}{2} \int u_{\varepsilon}^{2}(t) \zeta^{2} d x+\frac{1}{2} \iint\left|\nabla u_{\varepsilon}\right|^{2} \zeta^{2} d x d t+\iint \beta_{\varepsilon}\left(u_{\varepsilon}\right) u_{\varepsilon} \zeta^{2} d x d t \\
& \leq \frac{1}{2} \int u_{0 \varepsilon}^{2} \zeta^{2} d x+2 \iint u_{\varepsilon}^{2}|\nabla \zeta|^{2} d x d t .
\end{aligned}
$$

(iv) On the other hand, if we multiply by $\partial u_{\varepsilon} / \partial t$ we arrive at

$$
\begin{gathered}
\iint\left(\frac{\partial u_{\varepsilon}}{\partial t}\right)^{2} d x d t+\frac{1}{2} \int\left|\nabla u_{\varepsilon}\right|^{2} d x+\int \mathscr{B}_{\varepsilon}\left(u_{\varepsilon}\right) d x \\
=\frac{1}{2} \int\left|\nabla u_{0 \varepsilon}\right|^{2} d x+\int \mathscr{B}_{\varepsilon}\left(u_{0 \varepsilon}\right) d x,
\end{gathered}
$$

where $\mathscr{B}_{\varepsilon}$ is a primitive of $\beta_{\varepsilon}, \mathscr{B}_{\varepsilon}(s)=\int_{0}^{s} \beta_{\varepsilon}(\tau) d \tau$. Passing to the limit $\varepsilon \rightarrow 0$ the function $\mathscr{B}_{\varepsilon}(s)$ becomes $M$ times the Heaviside function, $M H(s)$, 
so that

$$
\int \mathscr{B}_{\varepsilon}\left(u_{\varepsilon}\right) d x \rightarrow M \lambda_{n}(\operatorname{supp}(u(t)))
$$

(by $\lambda_{k}(E)$ we denote the Lebesgue measure of a measurable set $E \subset \mathbf{R}^{k}$ ). Assuming that $\nabla u_{0}$ is in $L^{2}\left(\mathbf{R}^{n}\right)$ we get from (3.6) a uniform bound for the measure of the support of the solution of $(0.1)$ obtained in the limit.

(v) Observe finally that, due to the effect of the boundary condition, we have for a classical solution the following law for the evolution of the mass $\int u(x, t) d x$ :

$$
\frac{d}{d t} \int_{\Omega(t)} u(x, t) d x=-\lambda_{n-1}(\Gamma(t)),
$$

where $\Gamma(t)$ is the section of $\Gamma$ at time $t$. This law also holds for the weak solutions of Theorem 1.6. The result is easily derived from (3.2) and the convergence results of Sections 8-11.

\section{First gradient estimate}

Given a continuous and bounded initial function $u_{0} \geq 0$ with bounded gradient $\nabla u_{0}$ we construct for every $\varepsilon>0$ solutions $u_{\varepsilon}$ of the approximate problems $\left(\mathrm{P}_{\varepsilon}\right)$ which have bounded spatial gradient in $Q_{T}$ with a bound independent of $\varepsilon$.

We assume that $\beta_{\varepsilon}(s)=\varepsilon^{-1} \beta\left(\varepsilon^{-1} s\right)$, with $\beta$ as explained in the Introduction, though for convenience in the calculations we take as support the interval $[0,2]$, which is easily translated to $[0,1]$. We also assume that $\beta$ satisfies

$$
\begin{aligned}
& \text { there exists } M>0 \text { such that } \beta(s),-\beta^{\prime}(s) \leq M, \\
& \beta^{\prime}(s) \geq \beta(s) \text { for } 0<s<1 .
\end{aligned}
$$

This second condition is only a small additional restriction which we introduce because it simplifies the already lengthy calculations. We prove the following result.

Theorem 4.1. There exist approximations $u_{0 \varepsilon}$ of the data $u_{0}$ such that

$$
\sup _{Q}\left|\nabla u_{\varepsilon}\right| \leq K \max \left\{1, \sup \left|\nabla u_{0}\right|\right\}
$$

where $K \geq 1$ is a constant independent of $\varepsilon$.

Remark. The constant 1 in the second member of (4.2) accounts for the boundary condition $u_{\nu}=-1$.

Proof. Step 1. We take some initial data $u_{0 \varepsilon} \geq 0$ with bounded gradient (e.g., by mollification) and solve $\left(\mathrm{P}_{\varepsilon}\right)$. The solution $u_{\varepsilon}$ is positive in $Q$. We define the rescaled functions

$$
U_{\varepsilon}(x, t)=\frac{1}{\varepsilon} u_{\varepsilon}\left(\varepsilon x, \varepsilon^{2} t\right) .
$$

The $U_{\varepsilon}$ satisfy one and the same equation

$$
U_{t}=\Delta U-\beta(U),
$$


corresponding to problem $\left(\mathrm{P}_{\varepsilon}\right)$ with $\varepsilon=1$. They take on initial conditions $U_{\varepsilon}(x, 0)=\varepsilon^{-1} u_{0 \varepsilon}(\varepsilon x)$. Note that the gradients are preserved, i.e.,

$$
\nabla U_{\varepsilon}(x, t)=\nabla u_{\varepsilon}\left(\varepsilon x, \varepsilon^{2} t\right) .
$$

It is clear that we may replace $u_{\varepsilon}$ by $U_{\varepsilon}$ and $u_{0}$ by $U(x, 0)$ in (4.2). Therefore, we will work with rescaled solutions which satisfy one and the same equation. We will use a Bernstein technique. We introduce the change of variables (we drop the subscript $\varepsilon$ in $U$ and $v$ for convenience)

$$
U=\phi(v)
$$

for some increasing and $C^{2}$ function $\phi:[0, \infty) \rightarrow[0, \infty)$. After the change of variable equation (4.4) becomes

$$
v_{t}=\Delta v+\frac{\phi^{\prime \prime}(v)}{\phi^{\prime}(v)} v_{x}^{2}-\frac{\beta(\phi(v))}{\phi^{\prime}(v)}
$$

where $v_{x}=\nabla v$ denotes the spatial gradient of $v$ and the dot $\cdot$ will be used to denote scalar product in $\mathbf{R}^{n}$. Then

$$
v_{i t}=\Delta v_{i}+2 \frac{\phi^{\prime \prime}}{\phi^{\prime}} v_{x} \cdot v_{i x}+\left(\frac{\phi^{\prime \prime}}{\phi^{\prime}}\right)^{\prime} v_{i} v_{x}^{2}-\beta^{\prime}(U) v_{i}+\frac{\beta(U) \phi^{\prime \prime}(v)}{\phi^{\prime}(v)^{2}} v_{i},
$$

where the subscript $i$ denotes partial differentiation with respect to $x_{i}$. We now consider the quantity $w=v_{x}^{2}=\sum_{i} v_{i}^{2}$. It satisfies

$$
w_{t}=\Delta w+2 \frac{\phi^{\prime \prime}}{\phi^{\prime}} v_{x} \cdot w_{x}+2\left(\frac{\phi^{\prime \prime}}{\phi^{\prime}}\right)^{\prime} w^{2}+2\left\{\frac{\beta(U) \phi^{\prime \prime}}{\phi^{\prime 2}}-\beta^{\prime}(U)\right\} w-2 S
$$

where $S=\sum_{i, j} v_{i j}^{2} \geq 0$.

Step 2. Next we will make a special selection of $\phi$ and prove that if the maximum value of $w$ in $\bar{Q}_{T}$ is larger than a certain precise constant $k^{2}$ (which depends on $\beta$ and $\phi$ ), then it is necessarily attained at $t=0$.

The following additional properties of $\phi$ will be used: let $v_{1}$ and $v_{2}$ be the points determined by $\phi\left(v_{1}\right)=1$ and $\phi\left(v_{2}\right)=2$. There exist positive constants $a$ and $b$ such that in $I_{1}=\left\{0 \leq v \leq v_{1}\right\} \quad \phi$ is given by

$$
\phi(v)=e^{v}-1,
$$

while in $I_{2}=\left\{v_{1} \leq v \leq v_{2}\right\}$

$$
\left(\phi^{\prime \prime} / \phi^{\prime}\right)^{\prime} \leq-a
$$

and for $I_{3}=\left\{v>v_{2}\right\}$ we have

$$
\phi^{\prime}(v)=b .
$$

These conditions restrict the possible values of $v_{1}, v_{2}, a$ and $b$. Thus, $\phi\left(v_{1}\right)=1$ implies that $v_{1}=\log 2$. Observe also that with this construction $\phi$ is a convex function, so that $\phi^{\prime}$ is bounded below by $\phi^{\prime}(0)=1$ and above by $\phi^{\prime}\left(v_{2}\right)=b$. We will explain below the construction of a $\phi$ with these properties.

Going back to our argument about maxima of $w$, let us assume that the maximum of $w$ in a strip $\bar{Q}_{T}=\mathbf{R}^{n} \times[0, T]$ is attained at a certain point $\left(x_{0}, t\right)$ with $t>0$ and is larger than $k^{2}=2 M / a$. To arrive at a contradiction 
we argue as follows. At this point of interior maximum we necessarily have $w>0, w_{t} \geq 0, w_{x}=0$, and $\Delta w \leq 0$. It follows from (4.7) that

$$
E \equiv\left(\frac{\phi^{\prime \prime}}{\phi^{\prime}}\right)^{\prime} w+\left\{\frac{\beta(U) \phi^{\prime \prime}}{\phi^{\prime 2}}-\beta^{\prime}(U)\right\} \geq 0 .
$$

Let us analyze the quantity $E$ to conclude that it is indeed negative at such a point unless $w \leq k^{2}$. We do separate analyses in the regions $A=\{(x, t)$ : $0<U(x, t)<1\}, B=\{(x, t): 1 \leq U(x, t) \leq 2\}$, and $C=\{(x, t): 2<$ $U(x, t)<\infty\}$ corresponding respectively to $I_{1}, I_{2}$, and $I_{3}$.

- Region A. In this region we have $0<v<v_{1}$ and $\phi(v)=e^{v}-1$ so that $E$ reduces to $\beta(U) /(U+1)-\beta^{\prime}(U)$ which is negative by assumption (4.1b). Hence, no maximum can take place in this region.

- Region B. Since we have $\left(\phi^{\prime \prime} / \phi^{\prime}\right)^{\prime} \leq-a$ and $\beta,-\beta^{\prime} \leq M$ we get

$$
E<-a w+2 M \text {, }
$$

which is negative if $w>2 M / a$. We also have to check that no problem arises at the endpoints $v=v_{1}$ and $v=v_{2}$; we are safe because $\phi$ is $C^{2}$. Hence, $w$ is bounded above by $k^{2}=2 M / a$ in this region.

- Region C. Here $\beta \equiv 0$ so that $v_{t}=\Delta v$ and the gradient cannot attain an interior maximum.

Step 3. Assume now that the initial data have the gradient bound $\left|\nabla u_{0}\right| \leq L$. Since by assumption the function $\phi^{\prime}$ is bounded below away from 0 , by suitably smoothing $u_{0 \varepsilon}$ it is not difficult to construct approximations $u_{0 \varepsilon} \in C^{\infty}\left(\mathbf{R}^{n}\right)$ with $u_{0 \varepsilon}>0$ and $u_{0 \varepsilon} \rightarrow 0$ as $|x| \rightarrow \infty$ and such that

$$
\left|\nabla v_{0 \varepsilon}\right| \leq c L
$$

for some $c \geq \sup \left\{\left|\left(\phi^{-1}\right)^{\prime}(v)\right|\right\}$. By our result above we have

$$
\left|\nabla v_{\varepsilon}(x, t)\right|^{2} \leq \max \left\{c^{2} L^{2}, \frac{2 M}{a}\right\} .
$$

Using the fact that $\phi^{\prime}$ is bounded from above by $b$, we conclude that

$$
\left|\nabla u_{\varepsilon}(x, t)\right|=\left|\nabla U_{\varepsilon}\left(\frac{x}{\varepsilon}, \frac{t}{\varepsilon^{2}}\right)\right| \leq b \max \{c L, k\}, \quad k^{2}=\frac{2 M}{a},
$$

which ends the proof.

Step 4. Construction of $\phi$. We want to determine $\phi$ in $I_{2}=\left[v_{1}, v_{2}\right] . C^{2}$ continuity at $v=v_{1}$ implies that $\phi\left(v_{1}\right)=1, \phi^{\prime}\left(v_{1}\right)=\phi^{\prime \prime}\left(v_{1}\right)=2$. Putting $\left(\phi^{\prime \prime} / \phi^{\prime}\right)^{\prime}=-a(v)$ with $a(v) \geq a$, we have in $I_{2}$

$$
\frac{\phi^{\prime \prime}(v)}{\phi^{\prime}(v)}=1-A(v), \quad A(v)=\int_{v_{1}}^{v} a(s) d s .
$$

The interval $I_{2}$ continues until $\phi$ reaches the value 2 . Then, $C^{1}$-continuity of $\phi$ determines $b$. On the other hand, agreement of the second derivative implies that

$$
A\left(v_{2}\right)=\int_{v_{1}}^{v_{2}} a(s) d s=1
$$

which forces $\left(v_{2}-v_{1}\right) a$ to be smaller than 1. Since, in view of formula (4.12) we want $a$ to be as large as possible, we start at $v_{1}$ with $a(v)=a=2$. As 
long as this holds and $v-v_{1}<1 / 2$ we have in view of $(4.14) \phi^{\prime \prime}(v)>0$. Hence $\phi^{\prime}(v) \geq \phi^{\prime}\left(v_{1}\right)=2$ so that $\phi(v)=2$ can be reached in this interval, thus obtaining $v_{2}<v_{1}+1 / 2$. However, the agreement of the second derivative at $v_{2}$, condition (4.14), implies a modification $\phi:$ this can be done in a small interval near $v_{2}$ by taking $a(v)$ there to be as large as needed (this of course slightly decreases the value of $v_{2}$ ).

Remark. Observe that with this construction $c=\sup \left\{\left(\phi^{-1}\right)^{\prime}(v)\right\}$ appearing in formula $(4.12)$ is just $1 / \phi^{\prime}(0)=1$. On the other hand, integration of $\left(\phi^{\prime \prime} / \phi^{\prime}\right)^{\prime} \approx$ -2 in $\left[v_{1}, v_{2}\right]$ gives

$$
b=\phi^{\prime}\left(v_{2}\right) \approx \exp \left(v_{2}-\left(v_{2}-v_{1}\right)^{2}\right) \leq 2 \sqrt{e} .
$$

A careful construction of the functions $\beta$ and $\phi$ should allow to conclude that the best constant $K$ in estimate (4.2) is really 1 , but this entails complications that we do not feel we need at this moment.

\section{INTERIOR GRADIENT ESTIMATE}

We want to prove now that the result of the preceding section can be improved by making the gradient estimate independent of the gradient of the initial data, thus eliminating the need to assume that the initial data have such regularity. We will follow the notation and constructions of Section 4. Our assumption on the initial data is only an $L^{\infty}$-bound,

$$
0 \leq u_{0} \leq N
$$

and we will add $3 \varepsilon / 2$ to the approximate initial data $u_{0 \varepsilon}$. This will imply later a careful study of what free-boundary condition is satisfied. In order to avoid such a discussion, which we feel complicates the paper, we will leave the contents of this section as a remark and will not use the results subsequently.

In order to proceed we also ask that

$$
\beta^{\prime}(s) \geq 2 M+\beta(s) \text { for } 0<s<1 .
$$

Though $\beta$ is only Lipschitz continuous at $s=0$, no major problem arises with the solutions. For positive data solutions are positive for all times. We have

Theorem 5.1. There exists a constant $K>0$ such that

$$
\left|\nabla u_{\varepsilon}(x, t)\right| \leq K \max \{1, N / \sqrt{t}\} .
$$

$K$ depends only on $M$ and $n$.

Proof. Step 1. In performing the approximation we assume that the bound $N$ is satisfied by the initial functions $u_{0 \varepsilon}$, and hence by the solutions $u_{\varepsilon}$. Also, $u_{0 \varepsilon} \rightarrow 3 \varepsilon / 2$ as $x \rightarrow \infty$. We want to estimate the gradient $\nabla u_{\varepsilon}$ at a point $x_{0} \in \mathbf{R}^{n}$ and time $t_{0}>0$. By translation we may assume that $x_{0}=0$. Let us show to begin with that we can reduce any $t_{0}>0$ to the case $t_{0}=1$ by using the change of variables

$$
\tilde{u}(x, t)=\frac{1}{\lambda} u\left(\lambda x, \lambda^{2} t\right)
$$

with $\lambda=\sqrt{t_{0}}$. Observe that the gradient values are preserved, but $\|\tilde{u}\|_{\infty} \leq$ $N / \lambda=N / \sqrt{t_{0}}$. 
Step 2. Therefore, using the change of variables (4.3) we are concerned with the task of uniformly estimating $\nabla U_{\varepsilon}$ at a point $x_{\varepsilon}=0$ and time $t_{\varepsilon}=1 / \varepsilon^{2}$. The solution $U_{\varepsilon}$ is bounded from above by $N_{\varepsilon}=N / \varepsilon$. As for the bound from below our approximation satisfies $u_{0 \varepsilon} \geq 3 \varepsilon / 2$, hence $U_{0 \varepsilon} \geq 3 / 2$. Since $U_{\varepsilon}$ satisfies equation (4.4) and $\beta \leq M$, we can estimate $U_{\varepsilon}$ from below as follows:

$$
U_{\varepsilon}(x, t) \geq \frac{3}{2}-M t,
$$

so that $U_{\varepsilon}(x, t)$ is uniformly bounded below away from 0 for $0<t \leq \tau<$ $3 /(2 M)$.

We perform again a change of variables $U_{\varepsilon}=\phi\left(v_{\varepsilon}\right)$ as in (4.5), obtaining for $w=\left|\nabla U_{\varepsilon}\right|^{2}$ equation (4.7). We consider a point of maximum of the quantity

$$
z=\zeta w, \quad \zeta=\zeta(t)=t / t_{\varepsilon}=\varepsilon^{2} t,
$$

in the set $D_{\varepsilon}=\left\{(x, t): x \in \mathbf{R}^{n}, 0 \leq t \leq t_{\varepsilon}\right\}$. Since the solution is not zero, a maximum of $z$ is positive and attained at some point $(\hat{x}, \hat{t})$ with $0<\hat{t} \leq t_{\varepsilon}$. At such a point we have $w>0, w_{x}=0, \Delta w \leq 0$ and $z_{t} \geq 0$, i.e.

$$
w_{t} \geq-w\left(\zeta_{t} / \zeta\right)=-w / \hat{t} \text {. }
$$

Plugging this into (4.7) we get

$$
\left(\frac{\phi^{\prime \prime}}{\phi^{\prime}}\right)^{\prime} w+\left\{\frac{\beta(U) \phi^{\prime \prime}}{\phi^{\prime 2}}-\beta^{\prime}(U)\right\}+\frac{1}{\hat{t}} \geq 0,
$$

and multiplying by $\zeta$

$$
F=\left(\frac{\phi^{\prime \prime}}{\phi^{\prime}}\right)^{\prime} z+\left\{\frac{\beta(U) \phi^{\prime \prime}}{\phi^{\prime 2}}-\beta^{\prime}(U)\right\} \zeta+\varepsilon^{2} \geq 0 .
$$

We want to prove that there is a constant $K_{1}$ such that $z \leq K_{1}$ at $(\hat{x}, \hat{t})$. Assume for the moment that this is so. Then for every $x \in \mathbf{R}^{n}$

$$
w\left(x, t_{\varepsilon}\right) \leq z\left(x, t_{\varepsilon}\right) \leq z(\hat{x}, \hat{t}) \leq K_{1} .
$$

If $\phi^{\prime}$ is bounded above and below, $0<1 / c<\phi^{\prime}<b<\infty$ we obtain as in Section 4

$$
\left|\nabla u_{\varepsilon}(0,1)\right|=\left|\nabla U_{\varepsilon}\left(0, t_{\varepsilon}\right)\right| \leq b K_{1}=K,
$$

which ends the proof.

Step 3. In order to prove that $\max z \leq K_{1}$ we first have to select a suitable function $\phi$. It will be very similar to the one used in Section 4. If $N$ is not very large the only essential difference consists in the formula defining $\phi$ for $v \geq v_{2}$, i.e., $U \geq 2$, which becomes

$$
\phi^{\prime}(v)=b \exp \left(-\varepsilon^{2} v^{2}\right) .
$$

Observe that the only values of $v$ which matter are those such that $U=\phi(v) \leq$ $N / \varepsilon$. If $N$ is not very large, this upper bound is obtained for a value of $v$, say $v_{3}=C / \varepsilon$, by virtue of the following calculation:

$$
\phi\left(\frac{C}{\varepsilon}\right)=\phi\left(v_{2}\right)+b \int_{v_{2}}^{C / \varepsilon} e^{-\varepsilon^{2} v^{2}} d v \approx \frac{b}{\varepsilon} \int_{0}^{C} e^{-r^{2}} d r=\frac{N_{0}(C)}{\varepsilon} .
$$


This means that, according to formula $(5.11), \phi^{\prime}$ will have in the range $\left\{v_{2} \leq\right.$ $\left.v \leq v_{3}\right\}$ a value between

$$
\phi^{\prime}\left(v_{3}\right) \geq b \exp \left(-\varepsilon^{2}\left(C / \varepsilon^{2}\right)\right)=b \exp (-C)
$$

and $b=\phi^{\prime}\left(v_{2}\right)$. Consequently, $\phi^{\prime}=\phi_{\varepsilon}^{\prime}$ is bounded from above and below away from 0 independently of $\varepsilon$ in the range of definition of the solution $U_{\varepsilon}$. The assumption of the preceding step is fulfilled.

On the other hand, if $N$ is larger than $N_{0}$ we change the definition of $\phi$ by means of a contraction in the $v$-axis : $\tilde{\phi}(v)=\phi(\lambda v)$, applied to transform the interval $\left[0, v_{2}\right]$ into $\left[0, \tilde{v}_{2}\right], \tilde{v}_{2}=v_{2} / \lambda$, so that the slope at $\tilde{v}_{2}$ is $\tilde{b}=\lambda b$. Then we define $\tilde{\phi}$ for $v \geq \tilde{v}_{2}$ as

$$
\tilde{\phi}^{\prime}(v)=\tilde{b} \exp \left(-\varepsilon^{2} v^{2}\right) .
$$

If we put $\lambda=N / N_{0}$ we get for the point $v_{3}$ at which $\tilde{\phi}=N$ approximately the same point as before if $\varepsilon$ is small. Of course, $b$ has become $O(N)$. In the sequel we drop the tildes and assume that this change has been made if necessary.

Step 4. We can finally proceed with the analysis of the quantity $F$ at the point $(\hat{x}, \hat{t})$ and arrive at the conclusion $F<0$ if $z(\hat{x}, \hat{t})>K_{1}$, thus contradicting (5.8). We consider separately several regions.

- Region where $v>v_{2}$. As we said $v \leq v_{3}=O(1 / \varepsilon)$. By (5.11) we get $\left(\phi^{\prime \prime} / \phi^{\prime}\right)^{\prime}=-2 \varepsilon^{2}$. Besides, $\beta(U)$ and $\beta^{\prime}(U)$ are zero. Hence the expression for $F$ becomes $F=\varepsilon^{2}(1-2 z)$, which becomes negative if $z>1 / 2$.

- Region where $v_{1} \leq v \leq v_{2}$. With $\phi$ as in Section 4 we get

$$
F \leq-a z+2 M+\varepsilon^{2},
$$

which is negative if $z>K_{2}=\left(2 M+\varepsilon^{2}\right) / a$ (note that $0<\zeta \leq 1$ ). Therefore, $z \leq K_{2}$ in this region, accounting for the effect of the boundary condition $u_{\nu}=-1$.

- Region where $0<v<v_{1}$. Here the first term of $F$ disappears and the whole expression has to be controlled by the term $\beta^{\prime}(U) \zeta$. Using assumption (5.2) we are done. In particular, the term $\varepsilon^{2}$ is controlled by $\beta^{\prime}(U) \zeta$ because we have $U \leq 1$ so that, thanks to estimate (5.5), $\hat{t} \geq 1 /(2 M)$, namely $\zeta \geq \varepsilon^{2} /(2 M)$. No maximum happens in this region.

\section{REgULARITY IN TIME}

We show here that, as in the classical theory of the heat equation, Lipschitz continuity in $x$ implies Hölder continuity in $t$ with exponent $1 / 2$. We show in fact that away from the level $u=0$ the sequence of approximations of our problem discussed in Section 4 is uniformly Hölder continuous in time with exponent $1 / 2$ and constant independent of $\varepsilon$.

Theorem 6.1. There exists a constant $C>0$ depending only on $n$ such that for every $x \in \mathbf{R}^{n}$ and every $t_{2}>t_{1}>0$ we have

$$
\left|u_{\varepsilon}\left(x, t_{2}\right)-u_{\varepsilon}\left(x, t_{1}\right)\right| \leq C L\left(t_{2}-t_{1}\right)^{1 / 2}+C \varepsilon .
$$

Here $L \geq 1$ is a uniform bound for the spatial gradient of $u_{\varepsilon}$.

Proof. One part of the above inequality, even more, namely that $u_{\varepsilon}\left(x, t_{2}\right)-$ $u_{\varepsilon}\left(x, t_{1}\right)$ is bounded above by $C L\left(t_{2}-t_{1}\right)^{1 / 2}$, follows immediately from the 
fact that a solution of $\left(E_{\varepsilon}\right)$ is a subsolution of the heat equation, since it is a standard fact that Lipschitz continuity in $x$ implies Hölder continuity in $t$ with exponent $1 / 2$ for the latter equation.

Let us now find the estimate from below for $u_{\varepsilon}\left(x, t_{2}\right)-u_{\varepsilon}\left(x, t_{1}\right)$. Fix $x$ and $t_{1}$. It is enough to show that the inequality is true for $t_{2}-t_{1}>0$ small enough and when $u_{\varepsilon}$ is away from the action of the term $\beta_{e}\left(u_{\varepsilon}\right)$, say, $u_{\varepsilon}\left(x, t_{1}\right)=N \geq$ $5 \varepsilon>0$. It follows from the assumption that for $y \in \mathbf{R}^{n}$

$$
u_{\varepsilon}\left(y, t_{1}\right) \geq \max \{N-L|x-y|, 0\} .
$$

We are going to prove that $u_{\varepsilon}$ is bounded from below by $N / 2$ in a cylinder of the form $R(a, b)=B_{x}(a) \times\left(t_{1}, t_{1}+b\right)$ for some small $a$ and $b>0$. For this we recall the self-similar function (1.1), which is a solution of problem (P) for which the Maximum Principle holds, as proved in Corollary 1.4. We can choose $T$ so that its support at time $t_{1}$ will be strictly included in the support of the second member of $(6.2), B_{x}(N / L)$. We have shown in Section 1 that a small perturbation of this solution is in fact a subsolution of the approximate equation (0.4). Therefore, it will lie below $u_{\varepsilon}$. It follows that $u_{\varepsilon}(y, t) \geq N / 2$ if $(y, t) \in R(a, b)$ if we put $a=a_{0} N /(2 L)$ and $b=b_{0}(N / L)^{2}$ with $a_{0}$ and $b_{0}$ constants which depend only on $n$.

We thus have in $R(a, b)$ a solution of the heat equation taking values in the interval $N / 2 \leq u_{\varepsilon} \leq k N$ for some $k>1$. Hölder regularity follows now from standard theory in $R$. Moreover, by the rescaling

$$
\tilde{u}(x, t)=\frac{1}{N} u\left(\frac{x N}{L}, \frac{N^{2}}{L^{2}} t\right)
$$

we reduce this situation to the case $N=L=1$, and obtain a uniform coefficient $C>0$ which depends only on $n$ and the constants $a_{0}$ and $b_{0}$. Undoing the rescaling we see that the actual coefficient takes the form $C L$.

Remark. $C^{1 / 2}$-regularity in time is optimal at horizontal points of the free boundary as the explicit solution $U(x, t)$ of Section 1 shows.

\section{Passage to the limit $\varepsilon \rightarrow 0$}

We keep the assumption that $u_{0} \geq 0$ is bounded and Lipschitz continuous. We consider the family of solutions $\left\{u_{\varepsilon}(x, t)\right\}_{\varepsilon}$ to the approximate problems $\left(\mathbf{P}_{\varepsilon}\right)$, with $\varepsilon>0$ and with initial data which suitably approximate $u_{0}(x)$ as done in previous sections.

Theorem 7.1. Along a sequence the family $\left\{u_{\varepsilon}\right\}$ converges uniformly on compact subsets of $Q_{T}$ to a continuous function $u \geq 0$ of the Hölder class $C_{x, t}^{1,1 / 2}\left(Q_{T}\right)$. In its positivity set the function $u$ is a solution of the heat equation.

Proof. The functions $u_{\varepsilon}(x, t)$ form a uniformly bounded family in the space $C^{1,1 / 2}\left(Q_{T}\right)$. Therefore, up to selection of a sequence $\varepsilon_{j} \rightarrow 0$ we can pass to the limit and obtain a function

$$
u(x, t)=\lim _{\varepsilon_{j} \rightarrow 0} u_{\varepsilon_{j}}(x, t),
$$

and the limit is uniform on compact subsets of $\bar{Q}=\mathbf{R}^{n} \times[0, \infty)$. It follows that $u$ is a continuous and nonnegative function in $Q$, which belongs to the 
same space $C^{1,1 / 2}(Q)$. Moreover, on the positivity set

$$
\Omega=\{(x, t): u(x, t)>0\},
$$

which is an open subset of $Q, u$ is a smooth solution of the heat equation. Indeed, if $u\left(x_{0}, t_{0}\right)=c>0$, then, using the uniform convergence $u_{\varepsilon} \rightarrow u$ and the equicontinuity of the $u_{\varepsilon}$ we conclude that for every small $\varepsilon$ and in a small neighbourhood of $\left(x_{0}, t_{0}\right)$ we have $u_{\varepsilon}(x, t) \geq c / 2>\varepsilon$; hence $\beta_{\varepsilon}\left(u_{\varepsilon}\right)=0$. Hence, we have in the limit $u_{t}=\Delta u$.

The convergence can be precised by the following result that we shall need later.

Lemma 7.2. $\nabla u_{\varepsilon}$ converges to $\nabla u$ strongly in $L_{\text {loc }}^{2}$.

Proof. To make things simpler we will also assume that $u_{0} \in L^{2}\left(\mathbf{R}^{n}\right)$. Then the convergence takes place in $L^{2}$. We follow the idea of [BCN] for the elliptic problem. It is clear that along a subsequence $\nabla u_{\varepsilon}$ converges weakly to $\nabla u$. hence

$$
\iint|\nabla u|^{2} d x d t \leq \liminf _{\varepsilon \rightarrow 0} \iint\left|\nabla u_{\varepsilon}\right|^{2} d x d t .
$$

If we prove the converse inequality for every sequence $\varepsilon_{j} \rightarrow 0$ the result will follow. Now, multiplication of equation $\left(\mathrm{E}_{\varepsilon}\right)$ by $u_{\varepsilon}$ and integration in $Q_{T}$, $T>0$, gives

$$
\iint_{Q_{T}}\left|\nabla u_{\varepsilon}\right|^{2} d x d t=-\iint_{Q_{T}} u_{\varepsilon} \Delta u_{\varepsilon} d x d t=-\iint_{Q_{T}} u_{\varepsilon}\left(u_{\varepsilon, t}+\beta\left(u_{\varepsilon}\right)\right) d x d t .
$$

Since $u \beta(u) \geq 0$ we get

$$
\iint_{Q_{T}}\left|\nabla u_{\varepsilon}\right|^{2} d x d t \leq \frac{1}{2} \int u_{\varepsilon}^{2}(x, 0) d x-\frac{1}{2} \int u_{\varepsilon}^{2}(x, T) d x,
$$

so that in the limit

$$
\limsup _{\varepsilon \rightarrow 0} \iint_{Q_{T}}\left|\nabla u_{\varepsilon}\right|^{2} d x d t \leq \frac{1}{2} \int u^{2}(x, 0) d x-\frac{1}{2} \int u^{2}(x, T) d x .
$$

On the other hand, using the equation $u_{t}=\Delta u$, which is satisfied by the limit function $u$ in its positivity set, multiplying the equation $u_{t}=\Delta u$ by the function $u^{\delta}=\max \{u-\delta, 0\}$ and integrating we get

$$
\iint_{\{u>\delta, t \leq T\}}|\nabla u|^{2} d x d t=\frac{1}{2} \int\left(u^{\delta}\right)^{2}(x, 0) d x-\frac{1}{2} \int\left(u^{\delta}\right)^{2}(x, T) d x,
$$

so that in the limit

$$
\iint_{\{u>0, t \leq T\}}|\nabla u|^{2} d x d t=\frac{1}{2} \int u^{2}(x, 0) d x-\frac{1}{2} \int u^{2}(x, T) d x .
$$

Therefore,

$$
\limsup _{\varepsilon \rightarrow 0} \iint_{Q_{T}}\left|\nabla u_{\varepsilon}\right|^{2} d x d t \leq \iint_{Q_{T}}|\nabla u|^{2} d x d t,
$$

which completes the proof if $u_{0} \in L^{2}$. The general case can be obtained by an easy modification; cf. the local estimates (3.5).

We turn next to the equation globally satisfied by $u$, which depends on the limit behaviour of the term $\beta_{\varepsilon}\left(u_{\varepsilon}\right)$. 
Theorem 7.3. The term $\beta_{\varepsilon}\left(u_{\varepsilon}\right)$ converges in the limit to a finite measure $\mu$ supported on the free boundary $\Gamma$. For every $\phi \in C_{0}^{\infty}(\bar{Q})$ we have

$$
\iint_{\Omega} u\left(\phi_{t}+\Delta \phi\right) d x d t+\int u_{0}(x) \phi(x, 0) d x=\iint_{\Gamma} \phi d \mu .
$$

Proof. We start from the formula

$$
\iint_{Q} u_{\varepsilon}\left(\phi_{t}+\Delta \phi\right) d x d t+\int u_{0 \varepsilon}(x) \phi(x, 0) d x=\iint \beta_{\varepsilon}\left(u_{\varepsilon}\right) \phi d x d t,
$$

and pass to the limit $\varepsilon \rightarrow 0$ in all terms. The convergence of the first member follows from Theorem 7.1. As for the second member, we know from the a priori estimates of Section 3 that the family $\left\{\beta_{\varepsilon}\left(u_{\varepsilon}\right)\right\}_{\varepsilon}$ is bounded in $L_{\mathrm{loc}}^{1}\left(\bar{Q}_{T}\right)$, hence weakly convergent to a nonnegative measure $\mu$ in $\mathbf{R}^{n} \times[0, T]$, along a sequence $\varepsilon_{j} \rightarrow 0$ of course.

It is also clear that such a measure is concentrated on the free boundary $\Gamma$. On the one hand, it is not supported on $\{u>0\}$ because there $u_{t}=\Delta u$, as we explained in Theorem 7.1. The fact that $\mu$ does not charge the interior of $\{u=0\}$ is obtained by passing to the limit in the equation. In fact, if $\phi$ is a test function supported in the interior of the set $\{u=0\}$, we have

$$
\iint \beta_{\varepsilon}\left(u_{\varepsilon}\right) \phi d x d t=\iint u_{\varepsilon}\left(\phi_{t}+\Delta \phi\right) d x d t .
$$

Now, the second member tends to 0 as $\varepsilon \rightarrow 0$. Finally, we see that $\mu$ cannot be supported on the line $\{t=0\}$ by using the uniform continuity of the $u_{\varepsilon}$ near $t=0$ and formula (7.6). Formula (7.5) is now immediate.

\section{SOLUTIONS WITH SHRINKING SUPPORT}

We will end the construction of a weak solution of problem $(P)$ with the characterization of the boundary measure $\mu$. This is a delicate part. It so happens that the task is much easier when the support recedes, in other words when the flame advances. Therefore, we shall make suitable assumptions on $u_{0}$ that will enable us to control $u_{t}$, more precisely to show that it is nonpositive.

(H1): $\Omega_{0}$ is an open domain in $\mathbf{R}^{n}$ with $C^{2}$ boundary $\Gamma_{0}$ and $u_{0}$ is a $C^{2}$-function in $\bar{\Omega}_{0}$ which is positive in $\Omega_{0}$ and vanishes on $\Gamma_{0}$. We assume also that $\Delta u_{0} \leq 0$ in $\Omega_{0}$ and $0<a_{1} \leq\left|\nabla u_{0}\right| \leq a_{2}<1$ on $\Gamma_{0}$. If the domain is not bounded we need uniformity at infinity, in the sense that uniform bounds must exist for the curvature of $\Gamma_{0}$ and the $C^{2}$-norm of $u_{0}$ near $\Gamma_{0}$.

Under this assumption we have

Lemma 8.1. There exist admissible approximations $u_{\varepsilon}$ such that $\partial_{t} u_{\varepsilon} \leq 0$. Consequently, we get in the limit $\varepsilon \rightarrow 0$ a function $u$ such that $u_{t} \leq 0$. It follows that its positivity sets, $\Omega(t), t>0$, form a nonincreasing family of open sets. We also have

$$
\bigcup_{t>0} \Omega(t)=\Omega_{0} \text {. }
$$

Proof. (i) We construct smooth approximations $u_{\varepsilon}$ as described in previous sections and pass to the limit to obtain a 'solution' $u$. Differentiating in $t$ the approximate equation (0.4) we find for $v_{\varepsilon}=u_{\varepsilon, t}$ the equation

$$
v_{\varepsilon, t}=\Delta v_{\varepsilon}-\beta^{\prime}\left(u_{\varepsilon}\right) v_{\varepsilon} \text {. }
$$


The Maximum Principle applies to this equation. Therefore, if we approximate $u_{0}$ in such a way that $\Delta u_{0 \varepsilon} \leq \beta_{\varepsilon}\left(u_{0 \varepsilon}\right)$ we will obtain $\partial u_{0 \varepsilon} / \partial t \leq 0$, hence $\partial u_{\varepsilon} / \partial t \leq 0$ in $Q$. In the limit $\varepsilon \rightarrow 0$ we will get $u_{t} \leq 0$.

(ii.1) Therefore, everything depends on the approximation of the initial data $u_{0}$ with smooth, or at least Lipschitz-continuous, functions having the stated properties. To begin with, we consider for small $\varepsilon>0$ the set

$$
S_{\varepsilon}=\left\{x \in \Omega_{0}: 0<u<\varepsilon\right\} .
$$

By virtue of our assumptions, if $\varepsilon>0$ is small enough then $S_{\varepsilon}$ is a tubular neighbourhood of $\Gamma_{0}$. Moreover, there is a $C^{2}$ chart $g: \Gamma_{0} \times\left[0, \varepsilon_{0}\right) \mapsto \bar{\Omega}_{0}$ by means of which we represent a point $x \in S_{\varepsilon_{0}}$ in the form $x=g(y, z)$ with $y \in \Gamma_{0}, z \in\left(0, \varepsilon_{0}\right)$, and such that $g(y, 0)=y$ for $y \in \Gamma_{0}$. Observe that if $x=g(y, z)$, then we can view $y=\pi(x)$ as the projection of $x$ on $\Gamma_{0}$ given by this parametrization. Besides, the map $g$ can be chosen so that $z$ is the value of $u_{0}$, i.e.,

$$
g^{-1}(x)=\left(\pi(x), u_{0}(x)\right) \quad \text { in } S_{\varepsilon} .
$$

We may also consider the tubular neighbourhood extended to the range $-\varepsilon_{1}$ $<z<\varepsilon_{0}$, so that it includes a full neighbourhood of $\Gamma_{0}, S_{\left(-\varepsilon_{1}, \varepsilon_{0}\right)}=$ $g\left(\Gamma_{0} \times\left(-\varepsilon_{1}, \varepsilon_{0}\right)\right)$, lying on both sides of $\Gamma_{0}$. We will need the conditions $|\nabla z(x)| \leq a<1$ and $|\Delta z(x)| \leq C$ in $S_{\varepsilon_{1}, \varepsilon_{0}}$ for some constants $a \in\left(a_{2}, 1\right)$ and $C>0$. This can be done if $\varepsilon_{0}$ is small.

(ii.2) We now perform a one-dimensional construction as follows. We take a small $\delta>0$, precisely $(1+\delta) a^{2}<1$, and solve the problem

$$
\begin{aligned}
& f^{\prime \prime}(s)=(1+\delta) \beta(f(s)) \quad \text { for } z>0, \\
& f(0)=1, \quad f^{\prime}(0)=-1 .
\end{aligned}
$$

By integration we get the formula

$$
\left(f^{\prime}(s)\right)^{2}=1-2(1+\delta)\{\mathscr{B}(1)-\mathscr{B}(f(s))\},
$$

where $\mathscr{B}$ is a primitive of $\beta$ as in Section 3. As in the analysis of Lemma 1.3 the fact that $\left|f^{\prime}(0)\right|^{2}<2(1+\delta) \int \beta(u) d u$ implies that the solution $f(s)$ is decreasing until it reaches a positive minimum (which goes to 0 as $\delta \rightarrow 0$ ) at a point $s_{1}(\delta)$ (that is located at a distance which goes to infinity if $\delta \rightarrow 0$ ). By continuous dependence we may change a bit $f$ and obtain a new function $F(s)$ which solves

$$
F^{\prime \prime}(s) \leq(1+\delta) \beta(F)+\alpha F^{\prime} \quad \text { for } s>0,
$$

where $\alpha>0$ is a small number, and $F$ will still have the properties just discussed. Observe that $F$ depends on $\delta, F=F_{\delta}(s)$.

(ii.3) The next step consists in using this profile to modify the function $u_{0}$ in a neighbourhood of $\Gamma_{0}$ of the form

$$
S_{\varepsilon}^{*}=\{x=g(y, z):-c \varepsilon<z<\varepsilon\},
$$

with $0<\varepsilon<\varepsilon_{0}$ and $0<c \varepsilon<\varepsilon_{1}$. The correction is defined as follows:

$$
u_{0 \varepsilon}(x)=\varepsilon F_{\delta}\left(1-\frac{z}{\varepsilon}\right) \text {. }
$$

For $x \in \Omega_{0}-S_{\varepsilon}^{*}$ we keep the definition $u_{0 \varepsilon}(x)=u_{0}(x)$. We want $\nabla u_{0 \varepsilon}(x)=0$ at the outer boundary of the tube $S_{\varepsilon}^{*}$, where $z=-c \varepsilon$, which determines the 
constant $c$ as $c=s_{1}(\delta)-1$. On the outer set $U=\mathbf{R}^{n}-\left(\Omega_{0} \cup S_{\varepsilon}^{*}\right)$ we define the value of $u_{0 \varepsilon}$ as the constant $u_{0 \varepsilon}(x)=\varepsilon F\left(s_{1}(\delta)\right)$, which is $O(\varepsilon \delta)$. This completes the construction.

Observe that there is $C^{0}$ agreement of the different definitions on both boundaries of the tube $S_{\varepsilon}^{*}$, i.e., on $\Gamma_{+}$where $z=\varepsilon$, and on $\Gamma_{-}$where $z=$ $-c \varepsilon$. The gradient of $u_{0 \varepsilon}$ satisfies in $S_{\varepsilon}^{*}$ the formula

$$
\nabla u_{0 \varepsilon}(x)=-F^{\prime}(1-(z / \varepsilon)) \nabla z(x) .
$$

Therefore, since $z=u_{0}(x)$, this gradient is directed on $\Gamma_{+}$along the normal to the level line and satisfies, thanks to (8.5),

$$
\left|\nabla u_{0 \varepsilon}(x)\right|=\left|F^{\prime}(0) \nabla u_{0}(x)\right|=\left|\nabla u_{0}(x)\right| .
$$

Therefore, we obtain $C^{1}$ agreement on $\Gamma_{+}$. The same happens on $\Gamma_{-}$since the gradient vanishes. On the other hand, we have

$$
\Delta u_{0 \varepsilon}(x)=\frac{1}{\varepsilon} F^{\prime \prime}(1-(z / \varepsilon))\left|\partial_{x} z\right|^{2}-F^{\prime}(1-(z / \varepsilon)) \Delta_{x} z .
$$

It follows from (8.7) that for $\varepsilon$ small enough

$$
\Delta u_{0 \varepsilon}(x) \leq \frac{1}{\varepsilon}(1+\delta) \beta(F(1-(z / \varepsilon))) a^{2} \leq \beta_{\varepsilon}\left(u_{0 \varepsilon}(x)\right) .
$$

To end this construction step we observe that we can obtain convergence to 0 of the $u_{0 \varepsilon}$ outside of a set $\Omega_{0} \cup S_{0, \varepsilon^{*}}$ for any $\varepsilon^{*}>0$ by making $\delta \rightarrow 0$ as $\varepsilon \rightarrow 0$ but imposing that $\varepsilon c=\varepsilon\left(s_{1}(\delta)-1\right)$ goes to 0 . Moreover, we get $u_{0 \varepsilon} / \varepsilon \rightarrow 0$ in such a set. This last remark will be used in Section 10 (Lemma 10.4).

(iii) The fact that the family $\Omega(t)$ is nonincreasing is an immediate consequence of the formula $u_{t} \leq 0$. In order to show that $\Omega(t) \subset \Omega_{0}$ for every $t>0$ we only have to remark that for any compact set $K \subset \mathbf{R}^{n}$ we have $u_{\varepsilon}(x, t) \leq u_{0, \varepsilon}(x) \leq \varepsilon$ if $x \in K$ and $d\left(x, \Omega_{0}\right) \geq c \varepsilon$, where $c=c(K)$. Finally, in order to prove (8.1) it is enough to show that for every $x_{0} \in \Omega_{0}$ there exists a time $t_{0}=t\left(x_{0}\right)>0$ such that

$$
u\left(x_{0}, t\right)>0 \text { if } 0<t<t_{0} .
$$

Now, this property follows immediately from comparison with a small selfsimilar solution of the form (1.1) centered at $x_{0}$, using Corollary 1.4.

Remark. By approximation we can replace the condition $0<c_{1} \leq\left|\nabla u_{0}\right| \leq c_{2}<$ 1 by the less restrictive and more natural one, $0 \leq\left|\nabla u_{0}\right| \leq 1$.

The typical example consists in initial data $u_{0}$ with compact support and concave shape with suitable boundary slope, just like the self-similar solutions (1.1). Another interesting form is (for $n \geq 3$ )

$$
u_{0}(x)=A-\frac{b}{|x|^{n-2}} \text { for }|x|>a,
$$

where $a$ is determined by $u_{0}(a)=0$, hence $A a^{n-2}=b$. The boundary condition implies that $b \leq a^{n-1} /(n-2)$.

In practice we want a bit more, namely we would like to control $u_{t}$ by means of a directional derivative, say $u_{x_{1}}$. Since both $u_{t}$ and $u_{x_{1}}$ satisfy the linearized equation (8.1) we get 
Lemma 8.2. Let $\lambda \in \mathbf{R}$ and let $u_{0}$ satisfy $(\mathrm{H} 1)$. If moreover $u_{t}+\lambda u_{x_{1}} \leq 0$ at $t=0$, then the same is true for every $t>0$.

Having some control of the movement of the interface will greatly simplify the task of describing the boundary behaviour of the solution to $(\mathrm{P})$. In view of the above properties we will make the following assumption on our data, under which Lemma 8.2 will hold.

(H2): Besides the assumptions (H1) $u_{0}$ satisfies $\Delta u_{0}+\lambda\left|\nabla u_{0}\right| \leq 0$ in its positivity set for some constant $\lambda>0$.

A typical shape would be a function with compact support and strictly negative Laplacian in regions of big gradient, having also the right border jump for $\nabla u_{0}$. There are also interesting shapes with a hole and positive value as $x \rightarrow \infty$.

Corollary 8.3. If $(\mathrm{H} 2)$ holds for some $\lambda \in \mathbf{R}$, then the solution $u$ is nonincreasing along lines of the form $x(t)=x_{0}+\lambda$ te for every unit vector $\mathbf{e}$.

From Lemma 8.2 and Corollary 8.3 we conclude that

Corollary 8.4. Under assumption ( $\mathrm{H} 2)$ the null set $N$ of the solution $u$ has the following cone property: for every point $P_{0}=\left(x_{0}, t_{0}\right) \in N$ the forward cone

$$
K^{+}\left(P_{0}, \lambda\right)=\left\{(x, t):\left|x-x_{0}\right| \leq \lambda t, t \geq t_{0}\right\}
$$

is contained in $N$. Likewise, for every $P_{1}=\left(x_{1}, t_{1}\right)$ where $u$ is positive, the backward cone

$$
K^{-}\left(P_{1}, \lambda\right)=\left\{(x, t):\left|x-x_{0}\right| \leq \lambda t, 0 \leq t \leq t_{0}\right\}
$$

is contained in $P(u)$. Finally, the interface

$$
\Gamma=\partial\{(x, t): u(x, t)>0\}
$$

is described by a Lipschitz-continuous function $t=\Theta(x)$, with $x \in \Omega_{0}$.

\section{STUdY OF THE BOUNDARY MEASURE}

We resume the proof that in the limit of the approximations $u_{\varepsilon}$ we obtain a weak solution of Problem (P). We assume here the hypotheses of Section 7 plus the strict monotonicity condition $(\mathrm{H} 2)$ of the last section. As said at the end of Section 7 we only have to concentrate on the behaviour of the boundary measure. To begin with, let us review the notation. $\Omega=\{(x, t) \in Q: u(x, t)>$ $0\}$ denotes the positivity set of the solution $u, \Gamma=\partial \Omega$ its lateral boundary, i.e., the boundary in $Q$, and $\Omega(t), \Gamma(t)$ the respective sections at time $t$. Since $\Gamma$ is given by a Lipschitz continuous function $t=\Theta(x), x \in \Omega_{0}$, for a.e. point $(x, t) \in \Gamma$ there exists a normal vector $\nu=\nu(x, t)$ and a tangent plane to the surface. We have $\nu=\left(\nu_{1}, \ldots, \nu_{n}, \nu_{t}\right)$, which is proportional to $(-\nabla \Theta, 1)$. We denote by $d \Sigma$ the element of surface area on $\Gamma$, by $\alpha$ the angle formed by $\nu$ and the horizontal plane $\{t=$ constant $\}$, and by $d S=d \Sigma \cos \alpha$ the lateral projection of the area element, which typically enters calculations involving integration by parts with respect to the space variable. Indeed, $\cos \alpha=\left(\nu_{1}^{2}+\cdots+\nu_{n}^{2}\right)^{\frac{1}{2}}$.

In order to characterize the boundary measure we need some information on the convergence of the level lines of the approximate solutions. We consider the 
level lines $\gamma_{c}^{\varepsilon}$ consisting of the points $(x, t) \in Q$ where $u_{\varepsilon}(x, t)=c \varepsilon$. Due to Corollary 8.3 (and Corollary 8.4), time is given on these lines as a Lipschitz continuous function of space:

$$
t=\theta_{c}^{\varepsilon}(x), \quad x \in \mathbf{R}^{n} .
$$

By Sard's Theorem the level line $\gamma_{c}^{\varepsilon}$ is a $C^{\infty}$-surface for almost every $c>0$. We are interested in localizing the level lines $\gamma_{c}^{\varepsilon}$ near the free boundary $\Gamma$.

Lemma 9.1. Let $c \geq 1$ and $R>0$. For every $\delta>0$ there exists $\varepsilon_{0}>0$ such that $0<\varepsilon<\varepsilon_{0}$ implies

$$
\left|\theta_{c}^{\varepsilon}(x)-\Theta(x)\right| \leq \delta \quad \text { for } x \in \Omega_{0} \cap B_{R} .
$$

Proof. The estimate from below, $\theta_{c}^{\varepsilon}(x) \geq \Theta(x)-\delta$, is just a consequence of the continuity of $u$ and the uniform convergence of the $u_{\varepsilon}$, arguing as in Theorem 7.1.

We prove that the level lines $\gamma_{c}^{\ell}$ for $c \geq 1$ cannot be far above $\Theta(x)$ for $\varepsilon$ small by contradiction. Indeed, assume that there exist a sequence $\varepsilon_{j} \rightarrow 0$ and points $x_{j}$ such that

$$
\theta_{c}^{\varepsilon_{j}}\left(x_{j}\right) \geq \boldsymbol{\Theta}\left(x_{j}\right)+\delta .
$$

Let $t_{j}=\theta_{c}^{\varepsilon_{j}}\left(x_{j}\right)$. Then, by Lemma 8.4 there exists a cone $K_{j}$ with vertex at $\left(x_{j}, t_{j}\right)$ pointing backwards in time, such that $u_{\varepsilon_{j}} \geq c \varepsilon_{j}$ in $K_{j} \cap Q$. But then $\beta_{\varepsilon_{j}}\left(u_{\varepsilon_{j}}\right)=0$ and $u_{\varepsilon_{j}}$ satisfies in $K_{j}$ the heat equation. Next we observe that the sequence $x_{j}$ has a limit point, say $\bar{x}$, and the cones $K_{j}$ converge along the correspondent sequence to a cone $K$, whose vertex is located at distance at least $\delta$ over $\Gamma$. On $K$ the limit solution $u$ satisfies the heat equation. We are going to see that this is impossible. In fact $u$ is a nonnegative solution of the heat equation in $K$ which vanishes on the portion $K \cap\{t \geq \Theta(x)\}$ and is strictly positive on the complement of this set in $K$. Since both parts have nonempty interiors, the contradiction follows from the Strong Maximum Principle.

We proceed now to prove that the boundary measure $\mu$ is controlled by the area element of $\Gamma$ in space-time, $d \Sigma$.

Lemma 9.2. There exists a constant $C$ such that $d \mu \leq C d \Sigma$.

Proof. Let us take a smooth level line $\gamma_{c}^{\varepsilon}$ with $c>1$. Let $A_{\varepsilon}=\left\{u_{\varepsilon} \leq c \varepsilon\right\}$. Let $\phi$ be a test function in $C_{0}^{\infty}(Q)$ with support near a point of the free boundary $\Gamma$. Then

$$
\begin{aligned}
& \iint \beta_{\varepsilon}\left(u_{\varepsilon}\right) \phi d x d t=\iint_{A_{\varepsilon}} \beta_{\varepsilon}\left(u_{\varepsilon}\right) \phi d x d t=\iint_{A_{\varepsilon}}\left(\Delta u_{\varepsilon}-u_{\varepsilon, t}\right) \phi d x d t \\
& =\int_{\gamma_{c}^{\varepsilon}} \frac{\partial u_{\varepsilon}}{\partial \nu^{\varepsilon}} \phi d S^{\varepsilon}-\int_{\gamma_{c}^{\varepsilon}} u_{\varepsilon} \frac{\partial \phi}{\partial \nu^{\varepsilon}} d S^{\varepsilon}-\int_{\gamma_{c}^{\varepsilon}} u_{\varepsilon} \phi \nu_{t}^{\varepsilon} d \Sigma_{\varepsilon}+\iint_{A_{\varepsilon}} u_{\varepsilon}\left(\Delta \phi+\phi_{t}\right) d x d t .
\end{aligned}
$$

Here the $n$-dimensional measure $d S^{\varepsilon}$ denotes the lateral projection of the surface area element on $\gamma_{c}^{\varepsilon}$, i.e., $d S^{\varepsilon}=d \Sigma^{\varepsilon} \cos \alpha^{\varepsilon}$. Now, the last three terms tend to zero uniformly as $\varepsilon \rightarrow 0$. The first term in the second member is the only significant contribution. We can bound from above $\partial u_{\varepsilon} / \partial \nu^{\varepsilon}$ by the bound $L$ for the gradient. We thus obtain in the limit

$$
\iint \phi d \mu \leq c L \int_{\Gamma} d \Sigma .
$$

As a consequence of the Radon-Nikodym Theorem we get from this result the existence of a multiplier. 
Corollary 9.3. There exists a bounded, nonnegative function $\rho: \Gamma \rightarrow \mathbf{R}$ such that

$$
d \mu=\rho d \Sigma
$$

as measures in $Q$. Moreover, $0 \leq \rho(x, t) \leq L$.

The end of the existence proof consists in showing that the function $\rho$ equals $\cos \alpha$ almost everywhere on $\Gamma$. We recall that since $\Gamma$ is the graph of a Lipschitz continuous function $t=\Theta(x)$, almost every point $x \in \Omega_{0}$ is a point of differentiability of $\Theta$. At this stage we have to investigate more closely what happens at such points.

\section{Boundary Behaviour. ANAlysis OF Regular Points}

We perform in this section the local analysis near a nonhorizontal regular point of the free boundary $\Gamma$, where according to the basic example discussed in Section 1 we expect the solution to be well behaved. Indeed, we consider a point $P_{0}=\left(x_{0}, t_{0}\right)$ of $\Gamma$ where the free boundary is given by a differentiable surface and let $\nu_{0}$ be the exterior normal, which we assume is not directed along the time axis (a nonhorizontal point). After a rotation of coordinates we may assume the spatial projection of $\nu\left(P_{0}\right)=\nu_{0}$ points in the $x_{1}$ direction, i.e., $\nu_{0}=(\cos \alpha, 0, \ldots, 0, \sin \alpha)$ with $\cos \alpha>0$, i.e., $0<\alpha<\pi / 2$. The tangent plane $\Pi=\left\{(x, t):\left(x-x_{0}, t-t_{0}\right) \cdot \nu_{0}=0\right\}$ has equation

$$
x_{1}-x_{10}=(\operatorname{tg} \alpha)\left(t-t_{0}\right) \text {. }
$$

We prove the following result.

Theorem 10.1. Near $P_{0}$ the solution $u$ is given by the piecewise linear profile

$$
u(x, t)=\left[x_{1}-x_{10}-(\operatorname{tg} \alpha)\left(t-t_{0}\right)\right]_{+}+o\left(\left|x-x_{0}\right|+\left|t-t_{0}\right|\right) .
$$

The derivative $D u=\left(\nabla u, u_{t}\right)=\left(u_{x_{1}}, u_{x_{2}}, \ldots, u_{t}\right)$ at $P_{0}$, which amounts to $(-1,0, \ldots,-\operatorname{tg} \alpha)$, is taken along all nontangential interior directions. Moreover, we have the free-boundary condition

$$
u_{\nu}\left(P_{0}\right)=-1
$$

and the dynamic equation

$$
x_{1}^{\prime}\left(t_{0}\right)=u_{t}\left(P_{0}\right)=-\operatorname{tg} \alpha .
$$

Proof. We perform a linear scaling around $P_{0}$, i.e. we define

$$
u_{\lambda}(x, t)=\frac{1}{\lambda} u\left(\lambda\left(x-x_{0}\right), \lambda\left(t-t_{0}\right)\right),
$$

where $\lambda<1$, with the idea of passing to the limit $\lambda \rightarrow 0$ (blow-up). After translation we may assume that $P_{0}=(0,0)$. If we start with $u$ restricted to a ball $B_{R}=\{(x, t):|x|<R,|t|<R\}$, then $u_{\lambda}$ is defined at least in a box $B_{R / \lambda}$. Let $D_{\lambda}=\left\{(x, t) \in B_{R / \lambda}: u_{\lambda}(x, t)>0\right\}$. Because $\Pi$ is a tangent plane for every $R>0$ and $\varepsilon>0$ the boundary of $D_{\lambda} \cap B_{R}$ in $B_{R}$ can be confined in the region

$$
\Pi_{\varepsilon}=\left\{(x, t) \in B_{R}:\left|(x, t) \cdot \nu_{0}\right| \leq \varepsilon\right\}
$$

if $\lambda$ is small enough. Thus, in the limit $\lambda \rightarrow 0$ the region $D_{\lambda}$ converges to the half-space $\Pi^{-}=\left\{(x, t):(x, t) \cdot \nu_{0}<0\right\}$ and the convergence is uniform in 
balls $B_{r}$. In order to pass to the limit in $u_{\lambda}$ we first observe that the $\nabla u_{\lambda}$ are uniformly bounded. Moreover, using the fact that $\partial_{t} u_{\lambda} \leq 0$ and integrating we get a uniform bound for $\partial_{t} u_{\lambda}$ in $L_{\text {loc }}^{1}$. Therefore, along a sequence $\lambda_{j} \rightarrow 0$ we obtain the limit

$$
V=\lim _{\lambda_{j} \rightarrow 0} u_{\lambda_{j}}(x, t)
$$

in $L_{\text {loc }}$ and a.e., and $V$ is defined in $\mathbf{R}^{n+1}$. Necessarily, $V(x, t)=0$ in $\Pi^{+}$, i.e. for $(x, t) \cdot \nu_{0}>0$. Moreover

Lemma 10.2. There exists a function $c(t) \geq 0$ such that

$$
V(x, t)=c(t)\left[-x_{1}-(\operatorname{tg} \alpha) t\right]_{+} .
$$

Proof. Observe that the $u_{\lambda}$ satisfy in $D_{\lambda}$ the equation $\lambda \partial u_{\lambda} / \partial t=\Delta u_{\lambda}$. Now, we pass to the limit in the expression

$$
\iint\left(\lambda u_{\lambda} \phi_{t}+u_{\lambda} \Delta \phi\right) d x d t=0
$$

valid for any function $\phi \in C^{\infty}\left(\mathbf{R}^{n+1}\right)$ with support in $\Pi^{-}$if $\lambda$ is small enough. We get $\Delta_{x} V=0$ in $\Pi^{-}$. Since for fixed $t$ the function $V(x, t)$ is nonnegative and vanishes on the hyperplane $\Pi(t)$, the section of $\Pi$ at time $t$, there are only two options: either it is identically zero or it is positive with linear growth near the boundary (make a reflection around $x_{1}=0$ to obtain a harmonic function in the whole space which has linear growth, hence it is linear). This is just (10.7).

Lemma 10.3. The spatial gradient of $V$ at $P_{0}$, i.e., $c(t)$, is precisely 1 .

Proof. (i) Again we may take $P_{0}=(0,0)$. We consider the equation satisfied by the approximations $u_{\varepsilon}$, take a test function $\zeta$ supported near the point $P_{0}$, multiply the equation by $\zeta \partial_{k} u_{\varepsilon}$ and integrate to obtain (with the notation $u_{k}=\partial_{k} u$ and the convention of dropping the subscript $\varepsilon$ in $u_{\varepsilon}$; to avoid confusion we put $\lim u_{\varepsilon}=v$ )

$$
\iint u_{t} u_{k} \zeta d x d t=\iint \Delta u u_{k} \zeta d x d t-\iint \beta_{\varepsilon}(u) u_{k} \zeta d x d t
$$

Let us estimate the different terms when $\varepsilon \rightarrow 0$. To begin with, we have

$$
-\iint \beta_{\varepsilon}(u) u_{k} \zeta d x d t=-\iint \partial_{k}\left(\mathscr{B}_{\varepsilon}(u)\right) \zeta d x d t=\iint \mathscr{B}_{\varepsilon}(u) \zeta_{k} d x d t
$$

We claim that as $\varepsilon \rightarrow 0$ this quantity tends to

$$
\iint_{\{v>0\}} M \zeta_{k} d x d t
$$

where $M=\int \beta(s) d s=1 / 2$ (we use the letter $M$ to make more apparent the role played by the integral of $\beta$ ). This claim is not as immediate as it seems, and indeed it contains the important information that our approximations are correct. Let us postpone the proof to Lemma 10.4 and proceed. Since $u_{k}$ converges strongly in $L^{2}(Q)$ and $u_{t}$ weakly in $L^{2}(Q)$, we have

$$
\begin{aligned}
\iint u_{t} u_{k} \zeta d x d t & \rightarrow \iint v_{t} v_{k} \zeta d x d t=\iint_{\{v>0\}} v_{t} v_{k} \zeta d x d t \\
& =\iint_{\{v>0\}} v_{i i} v_{k} \zeta d x d t .
\end{aligned}
$$


Finally,

$$
\begin{aligned}
\iint \Delta u u_{k} \zeta d x d t & =-\iint u_{i} u_{k} \zeta_{i} d x d t-\iint u_{i} u_{k i} \zeta d x d t \\
& =-\iint u_{i} u_{k} \zeta_{i} d x d t+\frac{1}{2} \iint\left(u_{i}\right)^{2} \zeta_{k} d x d t
\end{aligned}
$$

which converges to

$$
\iint_{\{v>0\}}\left(\frac{1}{2}\left(v_{i}\right)^{2} \zeta_{k}-v_{i} v_{k} \zeta_{i}\right) d x d t
$$

We are using the summation convention in $i$. Summing up, we get

$$
\begin{aligned}
M \iint_{\{v>0\}} \zeta_{k} d x d t & =\iint_{\{v>0\}}\left(v_{i} v_{k} \zeta_{i}+v_{i i} v_{k} \zeta-\frac{1}{2}\left(v_{i}\right)^{2} \zeta_{k}\right) d x d t \\
& =\iint_{\{v>0\}}\left(\partial_{i}\left\{v_{i} v_{k} \zeta\right\}-\frac{1}{2} \partial_{k}\left\{\left(v_{i}\right)^{2} \zeta\right\}\right) d x d t
\end{aligned}
$$

(ii) We recall now that $v$ is approximately linear around $P_{0}=(0,0)$ and that $\nu_{k}=0$ for all spatial directions $k \neq 1$. We use the parabolic scaling given by

$$
v_{\lambda}(x, t)=\frac{1}{\lambda} v\left(\lambda x, \lambda^{2} t\right)=u_{\lambda}(x, \lambda t),
$$

which transforms solutions of the heat equation in $\Omega=\{v>0\}$ into new solutions in the corresponding domain $\Omega_{\lambda}=\left\{v_{\lambda}>0\right\}$, which tends as $\lambda \rightarrow 0$ to the hyperpane (in space-time) $\Pi_{1}=\left\{x_{1}=0\right\}$. Since the $v_{\lambda}$ have linear growth in $x$ and are nonincreasing in time, we pass to the limit

$$
\lim _{\lambda \rightarrow 0} v_{\lambda}(x, t)=\tilde{V}
$$

to find a nonnegative solution of the heat equation in the domain

$$
D_{1}=\left\{(x, t) \in \mathbf{R}^{n+1}: x_{1}<0\right\},
$$

with boundary value 0 on $\Pi_{1}$ and linear growth in $x$. This means that it is just the linear function $\tilde{V}(x, t)=c\left(-x_{1}\right)_{+}$, for some constant $c$ with $0 \leq c \leq L$. This we knew from Lemma 10.2. The convergence can be done in compact subsets $K$ of $D$, i.e., away from the boundary, to obtain

$$
\begin{array}{ll}
\partial_{k} v_{\lambda} \rightarrow \partial_{k} \tilde{V}=-\delta_{1 k} c & \text { in } K, \\
\partial_{t} v_{\lambda} \rightarrow \partial_{t} \tilde{V}=0 & \text { in } K .
\end{array}
$$

(Here $\delta_{i k}$ is Kronecker's delta.) We now observe that we may apply the scaling to formula (10.8), taking care of inserting $\zeta^{\lambda}(x, t)=\zeta\left(x / \lambda, t / \lambda^{2}\right)$ for some fixed $\zeta$, to get a formula valid for the $v_{\lambda}$. Suppose that we can pass to the limit $\lambda \rightarrow 0$ in all terms. Then we get with $k=1$

$$
M \int_{\Pi_{1}} \zeta d S=\iint_{\Pi_{1}}\left(c^{2} \zeta_{1}-\frac{1}{2} c^{2} \zeta_{1}\right) d x d t=\frac{c^{2}}{2} \int_{\Pi_{1}} \zeta d S,
$$

where $d S=d x_{2} \cdots d x_{n} d t=d \Sigma$ is the area element on $\Pi_{1}$. This proves that $c(0)^{2}=2 M=1$, as we wanted. 
(iii) The last step needs a better justification, since the convergence needed to pass to the limit in the second member of (10.8) was obtained only in the interior. A rigorous argument when $c>0$ is as follows: we observe that we can estimate a related integral (with $v=v_{\lambda}$ )

$$
\begin{aligned}
I_{\delta} & =\iint_{\{v>\delta\}}\left(\frac{1}{2} \partial_{k}\left\{\left(v_{i}\right)^{2} \zeta\right\}-\partial_{i}\left\{v_{i} v_{k} \zeta\right\}\right) d x d t \\
& =\iint_{\{v=\delta\}}\left(\frac{1}{2}\left(v_{i}\right)^{2} \nu_{k}-v_{i} v_{k} \nu_{i}\right) \zeta d \Sigma,
\end{aligned}
$$

where $\delta$ is a small positive constant independent of $\lambda$. This expression can be passed to the limit to obtain for $k=1$

$$
\iint_{\{v=\delta\}}\left(\frac{1}{2} c^{2}-c^{2}\right) \zeta d S
$$

which is what we wanted. We still have to estimate uniformly in $\lambda$ the remainder

$$
\iint_{\{\delta \geq v \geq 0\}}\left(\frac{1}{2}\left(v_{i}\right)^{2} \zeta_{k}-v_{i} v_{k} \zeta_{i}-v_{i i} v_{k} \zeta\right) d x d t
$$

The first and second terms are small for small $\delta$ because the gradient is bounded. For the third term we use the fact that $v_{t}=v_{i i} \leq 0$ to estimate

$$
\begin{aligned}
\left|I-I_{\delta}\right| & \cong \iint_{\{\delta \geq v \geq 0\}}\left|v_{t} v_{k}\right| \zeta d x d t \leq L \iint_{\{\delta \geq v \geq 0\}}\left(-v_{t}\right) \zeta d x d t \\
& \leq L N \int_{\{\delta \geq v \geq 0\} \cap\left\{t=t_{1}\right\}} v\left(x, t_{1}\right) d x,
\end{aligned}
$$

and this is small. The proof is complete for $c>0$.

The fact that $c \geq 0$ cannot be zero uses a similar but simpler argument. The first two terms in the second member of (10.8) disappear because $\nabla v_{\lambda}$ is bounded and converges to 0 in the interior of $D_{1}$, and for the third we again use the argument of integration in time.

(iv) Finally, the calculation for $c(\tau)$, with $\tau \neq 0$ is done in a similar way replacing formula $(10.9 \mathrm{a})$ by

$$
v_{\lambda}(x, t)=u_{\lambda}\left(x-(\operatorname{tg} \alpha) \tau \mathbf{e}_{1}, \lambda t+\tau\right) .
$$

Lemma 10.4. In the situation of Lemma 10.3 we have in any ball $B$

$$
\mathscr{B}_{\varepsilon}\left(u_{\varepsilon}\right) \rightarrow M \chi_{\{v>0\}},
$$

where $\chi_{\{v>0\}}$ denotes the characteristic function of the positivity set of $v$ in $B$. Proof. It is clear that near every point where $v>0$ we have uniform convergence of $u_{\varepsilon}(x)$ to $v(x)>c>0$, hence $\mathscr{B}_{\varepsilon}\left(u_{\varepsilon}\right) \rightarrow M$. This is the obvious part. The situation in the set $S=\{v=0\} \cap B$ is not at all so simple. First, we know that in any ball $B_{1}$ in $S$ we have convergence of $\beta_{\varepsilon}\left(u_{\varepsilon}\right)$ to 0 in $L^{1}$ (cf. (7.7)), and passing to a subsequence, almost everywhere convergence. Moreover,

$$
\iint_{B_{1}}\left|\nabla \mathscr{B}_{\varepsilon}\left(u_{\varepsilon}\right)\right| d x d t \leq L \iint_{B_{1}} \beta_{\varepsilon}\left(u_{\varepsilon}\right) d x d t \rightarrow 0,
$$

so that for a.e. $t>0$ we have $\mathscr{B}_{\varepsilon}\left(u_{\varepsilon}\right) \rightarrow k(t)$, a constant with respect to the space variable. Recall that we restrict the argument to the points of a ball 
contained in $S$. Since also $\beta_{\varepsilon}\left(u_{\varepsilon}\right) \rightarrow 0$ a.e. we have the alternative $k(t)=M$ or $k(t)=0$ in any such ball.

We want to eliminate the first possibility $k=M$. It is clear from the monotonicity of the $u_{\varepsilon}$ that the function $k(t)$ is monotone nonincreasing, so that, if we have $k=M$ on some nonnegligible set of times, then $k(t)$ must be 1 for all previous times. This argument can be extended by continuation to any connected component of the interior of $S$. Now, such connected components necessarily arrive at $t=0$. Here we use the assumption that the initial approximation is taken in such a way that $u_{0 \varepsilon} \rightarrow 0$ and $\mathscr{B}_{\varepsilon}\left(u_{0 \varepsilon}\right) \rightarrow 0$ a.e. in the complement of $\Omega_{0}$ (see proof of Lemma 8.1). We conclude that $k \equiv 0$ in $S$.

With these lemmas the proof of Theorem 10.1 is complete.

\section{Horizontal points. END OF THE EXISTENCE PROOF}

Taking into account the results of Section 10 for nonhorizontal regular points we go back to Corollary 9.3 and easily conclude that

Lemma 11.1. At a.e. nonhorizontal point we have $\rho=\cos \alpha$.

Proof. Just use formula (7.5) in a small box around $P_{0}$ and take into account the behaviour prescribed by Theorem 10.1 .

The analysis of horizontal points follows.

Lemma 11.2. At horizontal points $\rho=0$ a.e.

Proof. We make a linear rescaling at a point $P_{0} \in \Gamma$ where there exists a horizontal tangent plane $\Pi$. We assume that $P_{0}=(0,0)$ and then $\Pi=\{t=0\}$ and $\nu_{0}=(0, \ldots, 0,1)$. After making the linear scaling $(10.5)$ we have two options. Either (i) the functions $u_{\lambda}$ are uniformly bounded in a neighbourhood of $(0,0)$, or (ii) the limit function $U(x, t)$ becomes infinite at least for $t \leq-\delta<0$.

In the first case the spatial gradient, $\nabla u$, will tend to 0 as $(x, t) \rightarrow(0,0)$ along a nontangential cone in $\Pi_{-}=\{t<0\}$. Then we use again formula (7.5) to conclude that if $P_{0}$ is also a Lebesgue point for $\rho$ then $\rho=0$.

If (ii) holds then $u_{t}=-\infty$ at $P_{0}$, and then the level curves are very close to $\Pi$. This allows us to estimate that

$$
\iint \beta_{\varepsilon}\left(u_{\varepsilon}\right) d x d t \rightarrow 0
$$

This is based on the fact that $\beta_{\varepsilon}\left(u_{\varepsilon}\right) \approx 1 / \varepsilon$, while the increment $\Delta t \approx \Delta u / u_{t}<<$ $\varepsilon$ (here $A=$ increment). This completes the proof.

\section{ACKNOWLEDGMENT}

This work was partially done during visits of the first author to the Universidad Autónoma de Madrid, and of the second to the Institute for Advanced Study of Princeton. We are grateful to both institutions for their hospitality. We also thank the Institute for Mathematics and Its Applications, of Minneapolis, where both authors participated in the program on degenerate diffusions in the spring of 1991 . The research of the second author was partially supported by EEC Grant SC1-0019-C(TT). 


\section{REFERENCES}

[BCN] H. Berestycki, L. A. Caffarelli, and L. Nirenberg, Uniform estimates for regularization of free boundary problems, Analysis and Partial Differential Equations, Marcel Dekker, New York, 1990.

[BL] H. Berestycki and B. Larrouturou, $A$ semilinear elliptic equation in a strip arising in a two-dimensional flame propagation model, J. Reine Angew. Math. 396 (1989), 14-40.

[BLL] H. Berestycki, B. Larrouturou, and P. L. Lions, Multi-dimensional travelling-wave solutions of a flame propagation model, Arch. Rational Mech. Anal. 111 (1990), 33-49.

[BLN] H. Berestycki, B. Larrouturou, and L. Nirenberg, A nonlinear elliptic problem describing the propagation of a curved premixed flame, NATO Advanced Research Workshop on Mathematical Modelling in Combustion and Related Topics.

[BHS] M. Bertsch, D. Hilhorst, and C. Schmidt-Lainé, The well-posedness of a free-boundary problem arising in combustion theory, Preprint 21 Dép. Math., E. N. S. Lyon, France, 1989.

[BLS] C.-M. Brauner, A. Lunardi, and C. Schmidt-Lainé, Stabilité et instabilité des ondes stationnaires d'un problème de détonation à deux phases, C. R. Acad. Sci. Paris Sér. I Math. 311 (1990), 697-700.

[BuL] J. D. Buckmaster and G. S. S. Ludford, Theory of laminar flames, Cambridge Univ. Press, Cambridge, 1982.

[HH] D. Hilhorst and J. Hulshof, An elliptic-parabolic problem in combustion theory: convergence to travelling waves, Proc. Amer. Math. Soc. (to appear).

[S] D. S. Stewart, Transition to detonation in a model problem, J. Mech. Theor. Appl. 4 (1985), 103-137.

[SL] D. S. Stewart and G. S. S. Ludford, Fast deflagration waves, J. Mech. Theor. Appl. 3 (1983), 463-487.

[ZBLM] Ya. B. Zel'dovich, G. I. Barenblatt, V. B. Librovich, and G. M. Makhviladze, Combustion and explosions, Consultants Bureau, New York, 1985.

School of Mathematics, Institute for Advanced Study, Princeton, New Jersey 08540

Departamento de Matemáticas, Universidad Autónoma de Madrid, 28049 Madrid, SPAIN 